\title{
Perilipin 5 interacts with Fatp4 at membrane contact sites to promote lipid droplet-to- mitochondria fatty acid transport
}

Gregory E. Miner ${ }^{1}$, Christina M. So ${ }^{1}$, Whitney Edwards ${ }^{2}$, Laura E. Herring ${ }^{3}$, Rosalind A. Coleman $^{4}$, Eric L. Klett ${ }^{4,5}$, and Sarah Cohen ${ }^{1 *}$

${ }^{1}$ Department of Cell Biology and Physiology, University of North Carolina at Chapel Hill ${ }^{2}$ Department of Biology, University of North Carolina at Chapel Hill ${ }^{3}$ Department of Pharmacology, University of North Carolina at Chapel Hill ${ }^{4}$ Department of Nutrition, University of North Carolina at Chapel Hill ${ }^{5}$ Department of Medicine, University of North Carolina at Chapel Hill

*Correspondences: Sarah Cohen, sarahcoh@med.unc.edu

\section{Summary}

Cells adjust their metabolism by remodeling membrane contact sites that channel metabolites to different fates. Lipid droplet (LD)-mitochondria contacts change in response to fasting, cold exposure, and exercise. However, their function and mechanism of formation have remained controversial. We focused on perilipin 5 (Plin5), an LD protein that tethers mitochondria, to probe the function and regulation of LD-mitochondria contacts. We demonstrate that efficient LD-to-mitochondria fatty acid (FA) trafficking and ß-oxidation during starvation of myoblasts requires both phosphorylation of Plin5 and an intact Plin5 mitochondrial tethering domain. We further identified the acyl-CoA synthetase, Fatp4 (ACSVL4) as a novel mitochondrial interactor of Plin5. The C-terminal domains of Plin5 and Fatp4 constitute a minimal protein interaction capable of inducing organelle contacts. Our work suggests that starvation leads to phosphorylation of Plin5, lipolysis, and subsequent channeling of FAs from LDs to Fatp4 on mitochondria for conversion to fatty-acyl-CoAs and subsequent oxidation.

\section{Keywords}

Metabolism, organelles, lipid droplets, mitochondria, membrane contact sites, fatty acids, acylCoA, Perilipin 5, Fatp4 


\section{Introduction}

Cells must adjust their metabolism to respond to changing environmental or developmental cues. An emerging theme is that cells channel metabolites between cellular compartments at sites of close membrane apposition, called membrane contact sites (Prinz, Toulmay and Balla, 2019). These contact sites can be rapidly remodeled in response to signaling cues (Bohnert, 2020). In response to starvation, cytoplasmic lipases release fatty acids (FAs) from neutral lipids stored within the primary fat storage organelle, lipid droplets (LDs) (Zechner, Madeo and Kratky, 2017). FAs are then channeled into mitochondria, where they are oxidized. LD-mitochondria contacts increase in heart and muscle in response to fasting (Wang, Sreenivasan, et al., 2011) or exercise (Daemen, Van Polanen and Hesselink, 2018), and in tissue culture cells in response to starvation (Herms et al., 2015; Rambold, Cohen and Lippincott-Schwartz, 2015; Nguyen et al., 2017; Valm et al., 2017). These observations suggest that membrane contact sites may play a role in channeling lipids from LDs to mitochondria for oxidation. However, the function of contacts between LDs and mitochondria remains controversial. For example, in brown adipose tissue, LD-mitochondria contacts decreased during cold exposure. In this situation, LD-associated mitochondria promoted FA storage rather than oxidation (Benador et al., 2018). As a result, multiple roles for LD-mitochondria contact sites have been proposed. These roles include: (1) channeling FAs from LDs to mitochondria for oxidation; (2) channeling FAs or FA breakdown products from mitochondria to LDs to protect mitochondria from lipotoxicity; and (3) channeling ATP from mitochondria to LDs to promote neutral lipid synthesis (Wang and Sztalryd, 2011; Benador et al., 2019).

Perilipin 5 (Plin5) is one of only several proteins known to mediate LD-mitochondria contact sites (Schuldiner and Bohnert, 2017; Olzmann and Carvalho, 2018). Plin5 is a member of the conserved family of perilipin LD proteins. It is expressed in highly oxidative tissues including heart, skeletal muscle, and brown adipose tissue (Kimmel and Sztalryd, 2016). Superresolution microscopy has localized Plin5 specifically to sites of apposition between LDs and 
mitochondria (Gemmink et al., 2018). Plin5 consists of an N-terminal domain homologous to those of other perilipins, and a unique C-terminal region that recruits mitochondria to LDs (Wang, Sreenivasan, et al., 2011). Depletion or overexpression of Plin5 in mouse models has led to apparently contradictory results. Plin5-null mice have fewer LDs in heart and muscle (Kuramoto et al., 2012; Mason et al., 2014; Drevinge et al., 2016; Zheng et al., 2017), while overexpression of Plin5 in the heart leads to cardiac steatosis (Wang et al., 2013), suggesting a role for Plin5 in fatty acid sequestration. Consistent with these results, cardiomyocytes cultured from Plin5-deficient mice more actively oxidized FAs (Kuramoto et al., 2012). In contrast, Plin5 depletion led to reduced FA oxidation in liver (Montgomery et al., 2019), while overexpression of Plin5 in skeletal muscle or brown adipose tissue promoted oxidative gene expression (Bosma et al., 2013; Gallardo-Montejano et al., 2021). Some of these cell type-specific effects may be explained by the idea that phosphorylation of Plin5 by protein kinase A can act as a switch between lipolytic barrier and pro-lipolytic functions of Plin5 (Pollak et al., 2015; Keenan et al., 2021). Because of these contradictory phenotypes, it has been challenging to untangle the different potential functions of Plin5. In addition, the mitochondrial protein that Plin5 interacts with to mediate membrane contact sites has yet to be identified.

Here, we have carefully dissected the lipolytic barrier and tethering functions of Plin5. By expressing truncated and chimeric protein constructs, we identified a minimal Plin5 C-terminal domain necessary and sufficient to mediate LD-mitochondria contacts. Using fluorescent and radioactive FA pulse-chase assays, we found that both phosphorylation of Plin5 at serine 155 and an intact mitochondrial tethering domain are necessary for efficient channeling of FAs from LDs to mitochondria during starvation of C2C12 myoblasts. We further identified Fatp4 (ACSVL4), an acyl-CoA synthetase (Herrmann et al., 2001), as the mitochondrial binding partner of Plin5. Chimeric constructs containing the tether domains of Plin5 or FatP4 were sufficient to create artificial membrane contact sites at the LD-peroxisome or mitochondria- 
peroxisome interface. Finally, we show that both phosphorylation of Plin5 and Plin5-FatP4 interaction are necessary for efficient channeling of FAs from LDs to mitochondria.

\section{Results}

\section{Plin5 contains a C-terminal domain sufficient to promote LD-mitochondria contacts}

In vitro and in vivo studies have shown that Plin5 promotes FA storage in LDs and facilitates membrane contact sites between LDs and mitochondria (LD-mito contacts). The Nterminus of Plin5 contains a well characterized lipolytic barrier region that promotes FA storage and is conserved with other perilipins, while the unique C-terminal region is responsible for tethering LDs to mitochondria (Wang, Sreenivasan, et al., 2011). Previous studies demonstrated that expression of a chimeric protein fusing the C-terminal region of Plin5 (residues 396-463) to Plin2, a member of the perilipin family, conferred the ability to recruit mitochondria to LDs. Further, it was shown that partial truncation of the C-terminus reduces the ability of Plin5 to induce LD-mito contacts (Wang, Sreenivasan, et al., 2011). While the C-terminal region of Plin5 clearly mediates LD-mito contacts, the mitochondrial binding partner of Plin5 has not been identified.

To characterize the C-terminal region of Plin5, we sought to define the specific residues responsible for inducing LD-mito contacts. We first utilized I-TASSER (Roy, Kucukural and Zhang, 2010; Yang and Zhang, 2015; Yang et al., 2015), a protein structure prediction algorithm which compares primary sequence with known crystal structures, to predict the protein folding of Plin5. I-TASSER modeling suggested that Plin5 is composed of two separate structures, an Nterminal region homologous with the lipolytic barrier region of the perilipin family, and a Cterminal domain spanning residues 425-463, which we have termed the tether domain (Fig. 1A). The tether domain has no known closely related crystal structure. Therefore, we utilized QUARK (Xu and Zhang, 2012, 2013) de novo protein modeling to predict the structure of this domain. QUARK modeling predicts that the tether domain is composed of two a-helices with a 
hydrophobic pocket (Fig. 1B). Intriguingly, modeling also suggested this hydrophobic pocket may bind lipids, supporting a role in FA trafficking.

To further define the residues comprising the tether domain, we compared the ability of full-length mEmerald tagged Plin5 (Em-Plin5) to induce LD-mito contacts with a series of Plin5 constructs containing successive C-terminal truncations (Em-Plin5C $\Delta$ ), guided by our predicted structure (Fig. 1C). As expected, expression of Em-Plin5 in U2OS cells led to a drastic increase in LD-mito colocalization, indicating an increase in membrane contact sites between these organelles. In contrast, cells expressing even a 10-amino acid C-terminal truncation, Plin5C $\Delta$ (1-453), exhibited no increase in LD-mito colocalization (Fig. 1D,E). This suggests that both predicted $\alpha$-helices are essential for mitochondrial tether domain function. We also assessed whether the Plin5 tether domain regulates FA storage. We show that expression of Em-Plin5 and Em-Plin5C $\Delta$ constructs led to equivalent increases in LD area (Fig. 1F). These results suggest that Plin5 lipolytic barrier function is independent of LD-mito tethering.

To determine if the tether domain alone was sufficient to induce LD-mito contacts, we created Plin5 constructs lacking the lipolytic barrier region. To maintain LD localization, the lipolytic barrier region of Plin5 was replaced with the hairpin domain (HP) of Gpat4 (residues152-208), a minimal domain that allows targeting to LDs without affecting LD physiology (Wang et al., 2016) (Em-HP-Plin5C; Fig. 1G,H). Expression of only the HP domain (Em-HP) demonstrated efficient targeting to the LD, while having no effect on LD-mito contacts (Fig. 1I,J) or LD area (Fig. 1K). Strikingly, expression of Em-HP-Plin5C (396-463) significantly induced LD-mito colocalization. Serial truncations of the C-terminal region demonstrated that expression of the entire tether domain, Em-HP-Plin5C (425-463), is necessary and sufficient to induce LD-mito contacts (Fig. 1J). We also assessed if inducing LD-mito tethering promotes FA storage in the absence of the lipolytic barrier region. Consistent with the truncation experiments shown in Fig. 1F, our results show that expression of the Plin5 tether domain did not affect LD 
area (Fig. 1K). Together, these results show that removal of even 10 amino acids abrogates Plin5 mitochondrial tether function, but the whole tether domain (425-463) is required to induce LD-mito contacts.

We next tested the functions of the Plin5 lipolytic barrier and tether domains in a more oxidative cell type by expressing a subset of these truncation constructs in $\mathrm{C} 2 \mathrm{C} 12$ myoblast cells. As in U2OS cells, we found that the Plin5 lipolytic barrier domain was necessary and sufficient to increase LD area, while the tether domain was necessary and sufficient to increase LD-mito colocalization (Fig. S1A-C). We further examined the relationship between LD size, contacts with mitochondria, and LD movement. LDs are actively transported along microtubules, both at baseline and in response to changing nutritional conditions (Herms et al., 2015; Kilwein and Welte, 2019). We hypothesized that increasing LD-mito contacts by overexpressing Plin5 would result in reduced LD motion. Indeed, overexpression of full-length Plin5 significantly slowed LDs in both U2OS (Movies S1-S2) and C2C12 cells (Fig. S1D). Interestingly, expression of either the lipolytic barrier domain $(E m-P l i n 5 C \Delta)$ or tether domain $(E m-H P-P l i n 5 C)$ alone also slowed LD motion relative to control, with the tether domain having a larger effect (Movies S3S4, Fig. S1D). Notably full-length Plin5 slowed LDs to a greater extent than Em-Plin5C $\Delta$ or EmHP-Plin5C.This indicates that both larger LDs and LDs tethered to mitochondria have reduced motion on microtubules, and that Plin5 slows LDs via both mechanisms.

Taken together, our work demonstrates that the Plin5 tether domain, comprised of residues $425-463$, is necessary and sufficient to induce LD-mito contacts. Further, our data demonstrates that Plin5 lipolytic barrier activity is independent of LD-mito tethering, but that both lipolytic barrier and tethering domains of Plin5 affect LD motion.

Plin5 phosphorylation and LD-mito contacts drive ß-oxidation by increasing LD-tomitochondria FA trafficking 
Recent work has suggested that the formation of membrane contact sites, including those between LDs and mitochondria, function to facilitate the rapid transfer of molecules such as lipids. Given that Plin5-induced LD-mito contacts did not promote FA storage, we investigated whether these contacts instead promote trafficking of FAs from LDs to mitochondria under starvation conditions. To assess LD-to-mitochondria FA trafficking, we used an established pulse-chase assay that visualizes FA trafficking using the fluorescent FA analog Bodipy 558/568 C 12 (Red C12) (Fig. 2A) (Rambold, Cohen and Lippincott-Schwartz, 2015). C2C12 myoblasts were treated with trace amounts $(5 \mu \mathrm{M})$ of Red C12 overnight to allow accumulation in LDs, followed by a $1 \mathrm{hr}$ incubation in complete medium (CM) to remove excess label. Red C12 colocalization with LDs and mitochondria was then monitored immediately before (Fig. 2B, CM) and every 30 min following incubation in Hank's Balanced Salt Solution (HBSS) for 4 hrs. Following incubation in HBSS, cells showed dramatic relocalization of FAs from LDs to mitochondria (Fig. 2B).

Protein kinase $A(P K A)$, a crucial regulator of lipolysis, is activated through phosphorylation during fasting (Pollak et al., 2015). Recent studies have shown that Plin5 can also be phosphorylated at Serine 155 by PKA, ablating lipolytic barrier activity and thereby allowing lipolysis (Wang, Bell, et al., 2011; Keenan et al., 2021). Given that lipolysis is required for LD-to-mitochondria FA trafficking, we created phosphomimetic (S155E) variants of our full length and truncated Plin5 constructs to investigate the contribution of LD-mito contacts to FA trafficking when lipolysis is active. Previous studies found that expression of Plin5 constructs with the same mutation replicated effects of PKA-mediated Plin5 phosphorylation, including changes in cellular localization and transcription (Gallardo-Montejano et al., 2016; Najt et al., 2020). However, the ability of this phosphomimetic mutation to ablate lipolytic barrier activity has not previously been demonstrated. We first assessed whether expression of our phosphomimetic constructs failed to promote FA storage. As anticipated, cells expressing Plin5 
showed increased storage of FAs in LDs in response to a Red C12 pulse; in contrast, cells expressing either Plin5 S155E or Plin5CA S155E showed no significant increase in FA storage, indicating that our phosphomimetic mutation inactivated lipolytic barrier activity (Fig. 2C). We attribute decreased FA storage to altered lipolytic barrier activity, as both Plin5 S155E and Plin5C $\Delta$ S155E constructs localized appropriately to LDs and affected LD-mito contacts equivalent to Plin5 and Plin5C $\Delta$, respectively (Fig. S2A-B).

We then assessed the ability of our Plin5 constructs to affect FA trafficking during starvation. Cells overexpressing Plin5 failed to traffic FAs from LDs to the mitochondria after 4 hrs in HBSS, suggesting the lipolytic barrier region was not phosphorylated during the time scale of our assay and thus lipolysis could not occur. Strikingly, cells expressing Plin5 S155E showed a dramatic increase in FA trafficking to the mitochondria in response to starvation. In contrast, Plin5C $\Delta$ S155E-expressing cells, which have active lipolysis but do not induce LD-mito contacts, showed no significant change in FA trafficking compared to control cells during HBSS incubation (Fig. 2D). These data demonstrate that both Plin5 phosphorylation and LD-mito contacts function to enhance LD-to-mitochondria FA trafficking under starvation conditions.

To investigate whether the observed effects of Plin5 expression on FA trafficking led to changes in FA esterification and $\beta$-oxidation, we used a modified version of our pulse-chase assay described above by using trace amounts $(17 \mu \mathrm{M})$ of a radioactive FA analog, [1$\left.{ }^{14} \mathrm{C}\right]$ oleate. Using thin-layer chromatography (TLC) we confirmed incorporation of $\left[1-{ }^{14} \mathrm{C}\right]$ oleate into LD-specific neutral lipids, including triacylglycerol (TAG), diacylglycerol (DAG) and cholesterol esters (CE), with TAG being the most abundantly labeled species (Fig. 2E). Expression of Plin5 led to increased incorporation of $\left[1-{ }^{14} \mathrm{C}\right]$ oleate into LD-specific neutral lipids, while Plin5 S155E did not (Fig. 2E). This confirms the ability of our phosphomimetic construct to ablate Plin5 lipolytic barrier activity. Intriguingly, we observed that overexpression of Em-Plin5 led to a significant increase in incorporation of $\left[1-{ }^{14} \mathrm{C}\right]$ oleate into phosphatidylserine (PS). 
Membrane contact sites between ER and mitochondria facilitate PS transport into mitochondria, where PS is subsequently converted to phosphatidylethanolamine (PE) (Kannan et al., 2017). By promoting LD-mito contacts, Plin5 overexpression may indirectly reduce ER-mitochondria contact sites and thereby inhibit the transport and subsequent conversion of PS to PE. To assess $\beta$-oxidation, we measured the amount of ${ }^{14} \mathrm{C}$ acid soluble molecules (ASM) released from cells following nutrient starvation. Mirroring our FA trafficking assay, we found that expression of Em-Plin5 did not change $\beta$-oxidation compared to control. In contrast, expression of Em-Plin5 S155E significantly increased $\beta$-oxidation relative to control and Em-Plin5. Finally, expression of truncated phosphomimetic Plin5C $\Delta$ S155E failed to increase $\beta$-oxidation relative to control and showed reduced $\beta$-oxidation compared to Plin5 S155E (Fig. 2F).

Previous work has shown that over extended time periods, overexpression of Plin5 S155E can promote transcription of genes that mediate mitochondrial biogenesis and oxidative function, including carnitine palmitoyltransferase I (Cpt1) (Gallardo-Montejano et al., 2016). Increased protein expression of Cpt1 has the potential to affect FA transport into mitochondria and $\beta$-oxidation. However, on the time scale of our assays (cells transfected for $24 \mathrm{hrs}$ followed by 4 hr starvation), expression of Plin5 S155E did not significantly increase levels of Cpt1 protein (Fig. S2D). Therefore, the effects of expressing Plin5 S155E in our assays can be attributed to protein-protein interactions and LD-mitochondrial tethering, and not to transcriptional effects.

Together, these data demonstrate that Plin5-induced LD-mito contacts enhance the trafficking of FAs from LDs to mitochondria during starvation, leading to increased $\beta$-oxidation. This activity is dependent on phosphorylation of the Plin5 lipolytic barrier region, suggesting that Plin5 mediates the metabolic shift from glycolysis to $\beta$-oxidation by regulating both lipolysis and FA trafficking. 


\section{The Plin5 tether domain interacts with acyl-CoA synthetase Fatp4}

Our studies thus far have established a physiological consequence of LD-mito contacts induced by the tether domain of Plin5. However, the mechanisms by which these contacts form remains unclear. We hypothesized that the tether domain of Plin5 interacts with one or more outer mitochondria membrane (OMM) proteins. To identify protein interactions specific to the tether domain of Plin5 we performed affinity purification-mass spectrometry (AP-MS) with cells expressing either Em-Plin5 or Em-Plin5C $\Delta$. To understand the contribution of the two predicted a-helices (Fig. 1B) to protein binding, we utilized Em-Plin5C $\Delta$ constructs lacking either a single helix (Em-Plin5C $\Delta(1-443))$ or both (Em-Plin5C $\Delta(1-424))$. Em-Plin5 and Em-Plin5C $\Delta$ protein complexes were affinity purified from transfected U2OS cells using a high-affinity nanobody for GFP (GFP-Trap). As a control, affinity purification was performed using cells transfected with mEmerald alone (Em). Affinity-purified proteins were then analyzed by LC-MS/MS on a QExactive HF mass spectrometer. Two independent biological replicates were analyzed in duplicate. Proteins were considered putative Plin5 interactors if they showed 2-fold enrichment over control and were detected by at least 2 peptides in both biological replicates. Putative interactors were then analyzed using SAINT (Significance Analysis of INTeractome) (Choi et al., 2012) to identify Plin5 interactors with high confidence.

A total of 59 putative Plin5 interactors passed our statistical cutoffs (Supplementary Table 1). Of these, 24 showed decreased abundance in both of our affinity-purified Plin5 $\mathrm{C} \Delta$ protein complexes (Fig. 3A,B) suggesting they interact specifically with the tether domain of Plin5. For most tether-specific interactors (19/24), we found that full truncation of the tether domain led to a 2-fold or greater reduction in protein binding relative to partial truncation, suggesting the a-helix spanning residues $425-443$ may be primarily responsible for proteinprotein interactions. We identified two proteins that were of particular interest due to their mitochondrial localization and role in in FA metabolism: Fatp4 and Acot9. Acot9 is primarily 
localized to the inner mitochondrial membrane (Steensels et al., 2020). Therefore, we deemed it unlikely to facilitate organelle tethering, though this data may suggest the formation of a complex spanning mitochondrial membranes. Fatp4 is an OMM bifunctional Acyl-CoA synthetase (ACSVL) that has FA transport and acyl-CoAs synthetase activity, with substrate preference towards C16-C24 FAs (Hall et al., 2005). Fatp4 has also been implicated in promoting $\beta$-oxidation through re-esterification of long chain FAs following lipolysis (Jia, Moulson, et al., 2007; Jia, Pei, et al., 2007; Lobo et al., 2007). Related ACSL proteins such as ACSL1 have been shown to associate with LD tethering proteins such as Snap23 (Young et al., 2018), supporting a potential role in mediating LD-mito contacts. Further, exercise increases expression of both Plin5 (Shepherd et al., 2013) and Fatp4 (Jeppesen et al., 2012), which may be primarily responsible for the observed increase in LD-mito contacts (Tarnopolsky et al., 2007; Devries et al., 2013).

We confirmed that Fatp4 interacts with the tether domain of Plin5 by western blot analysis of our affinity-purified Plin5 complexes (Fig. 3C). While it did not appear in our AP-MS screen, Mitofusin 2 (Mfn2) has recently been implicated as a potential LD-mito tether through interaction with Plin1 (Boutant et al., 2017). Because the lipolytic barrier domains of Plin1 and Plin5 are conserved, we tested whether Plin5 interacts with Mfn2. We show that Mfn2 is in fact a novel Plin5 interactor; however, in contrast to Fatp4, Mfn2 binding to Plin5 does not decrease upon truncation of the tether domain (Fig. 3C,D). This finding is consistent with Mfn2 interacting with both Plin1 and Plin5 via the homologous lipolytic barrier region.

\section{Plin5 and Fatp4 drive membrane contact site formation}

Given that Fatp4 interacts specifically with the Plin5 tether domain, we next investigated whether these proteins constitute a minimal protein interaction sufficient to drive membrane contact site formation. If these proteins constitute a minimal membrane tether they should be capable of inducing contact site formation in the absence of additional LD or mitochondrial 
proteins. To test this possibility, we first assessed whether recruitment of the Plin5 tether domain to peroxisomes could induce peroxisome-mitochondria contact sites. We adapted our LD-targeted construct design by replacing the HP domain with the peroxisomal membrane protein targeting sequence (residues 1-33) of Pex3 (Pex) to localize the Plin5 tether domain to peroxisomes (Pex-Em-Plin5C) (Fig. 4A-B). Expression of control constructs containing only mEmerald tagged Pex (Pex-Em) in U2OS cells showed efficient recruitment to peroxisomes while having no apparent effect on peroxisome-mitochondria colocalization. In contrast, expression of Pex-Em-Plin5C did cause a significant increase in peroxisome-mitochondria colocalization (Fig. 4C-D). Expression of Pex-Em-Plin5C did not affect peroxisome area relative to control (Fig. S3A). These results suggest that the ability of the Plin5 tether domain to drive membrane contact site formation is not dependent on the unique membrane architecture of the LD.

We next assessed whether HP-Em-Plin5C can induce contact sites with Fatp4 relocalized to the peroxisomal membrane. Fatp4 is an integral membrane protein containing a large cytosolic C-terminal domain (156-643) (Cho et al., 2020), which we hypothesized contained the Plin5 interaction site. For these experiments, we created constructs which localize the C-terminal domain of Fatp4 (Fatp4C) to the peroxisomal membrane (Pex-BFP-Fatp4C) (Fig. 4E,F). Co-expression of Em-HP-Plin5C and Pex-BFP-Fatp4C showed an approximately 2-fold increase in LD-peroxisome colocalization compared to controls transfected with only membrane targeting sequences (Em-HP and Pex-BFP). In contrast, expression of Em-HP-Plin5C or PexBFP-Fatp4C alone did not increase LD-peroxisome colocalization (Fig. 4G,H). Expression of these constructs either alone or together did not affect LD or peroxisome area (Fig. S3B,C). Together, these data suggest that the Plin5 tether domain and Fatp4 C-terminal domain form a minimal protein interaction sufficient to drive membrane contact sites. Further, the ability to 
relocalize either protein while retaining contact site induction suggests additional resident LD or mitochondrial proteins are not required for this activity.

\section{Loss of Fatp4 impairs Plin5 induced LD-mito contacts and FA trafficking}

Recent work to characterize membrane contact sites has shown that many utilize multiple tethers, and loss of a single tether may not drastically alter contact site formation (Prinz, 2014). While we have shown that Plin5 and Fatp4 can interact to induce membrane contact sites, we next tested whether Fatp4 is required for Plin5-driven LD-mito contacts. We assessed the ability of Plin5 to induce LD-mito contacts in cells depleted of Fatp4. Fatp4 expression in C2C12 myoblasts was inhibited by siRNA transfection (Fatp4 siRNA), leading to a $>70 \%$ reduction in Fatp4 protein abundance relative to non-targeting siRNA (NT siRNA) (Fig. S4A,B). Expression of Em-Plin5 in Fatp4-knockdown cells showed an $~ 50 \%$ reduction in LD-mito contacts relative to NT siRNA controls (Fig. 5A,B). The inability of Fatp4-knockdown to fully ablate Em-Plin5 induction of LD-mito contacts suggests there may be additional Plin5 binding partners on the mitochondrial membrane that facilitate LD-mito contacts. Alternatively, these results may be due to incomplete knockdown of Fatp4 as $10-30 \%$ of Fatp4 remained following Fatp4 siRNA knockdown. In contrast, loss of Fatp4 did not appear to affect Plin5 lipolytic barrier function, as expression of Em-Plin5 increased LD area in Fatp4-knockdown cells comparable to NT siRNA treated cells (Fig. 5C).

Finally, we investigated whether the loss of Fatp4 alters the ability of Plin5 to facilitate LD-to-mitochondria FA trafficking during starvation using the pulse-chase assay shown in Fig. 2A. After Red C12 pulse, Fatp4-knockdown cells transfected with phosphomimetic Plin5 S155E stored a similar amount of RedC12 within LDs compared to NTsiRNA treated cells (Fig. 5D). Upon starvation however, Fatp4-depleted cells expressing Plin5 S155E were unable to efficiently traffic FAs to mitochondria (Fig. 5E). Interestingly, loss of Fatp4 completely ablated Plin5 S155E-induced FA trafficking while only partially preventing LD-mito contacts (Fig. 5B,E). 
This indicates that Fatp4 not only serves as a LD-mito tether but may also directly facilitate FA trafficking through its ACSL activity. Taken together, our data demonstrate that the tether domain of Plin5 interacts with Fatp4 to induce LD-mito contacts. Further, these Plin5-induced membrane contact sites facilitate trafficking of FAs from LDs to mitochondria during nutrient starvation, promoting $\beta$-oxidation.

\section{Discussion}

We have focused on the LD protein Plin5 to investigate the function and mechanism of formation of LD-mito contact sites. We used predictive modeling and expression of truncated and chimeric protein constructs to precisely identify a minimal Plin5 C-terminal domain required for tethering to mitochondria (amino acids 425-463; Fig. 1). The creation of a truncation construct lacking this domain allowed us to identify an acyl-CoA synthetase, Fatp4, as the mitochondrial binding partner of Plin5 by co-immunoprecipitation/mass spectrometry (Fig. 3). Chimeric constructs containing the C-terminal tether domains of Plin5 and Fatp4 were sufficient to create membrane contacts at the LD-peroxisome or mitochondria-peroxisome interface (Fig. 4). We used fluorescent and radioactive FA pulse-chase assays to test the function of LD-mito contacts during starvation of $\mathrm{C} 2 \mathrm{C} 12$ myoblast cells. We found that phosphorylation of Plin5 at serine 155, an intact Plin5 mitochondrial tethering domain, and expression of Fatp4 are all necessary for efficient channeling of FAs from LDs to mitochondria and subsequent $\beta$-oxidation in response to starvation (Figs. 2 and 5). We propose that during starvation: (1) PKA phosphorylates Plin5 at serine 155 (Keenan et al., 2021); (2) phosphorylation of Plin5 promotes lipolysis of triacylglycerol and release of FAs (Wang, Bell, et al., 2011; MacPherson et al., 2013; Pollak et al., 2015); (3) released FAs are channeled from LDs to mitochondria at membrane contact sites, where Fatp4 converts FAs to fatty-acyl-CoAs, allowing; (4) transport into mitochondria for $\beta$-oxidation (Fig. 5F). 
Our de novo modeling of the Plin5 tether domain suggests that this domain contains a hydrophobic pocket that may bind lipids (Fig. 1B). Plin5 has previously been shown to bind lipids, with lipid binding regulated by phosphorylation (Najt et al., 2020). FA binding is predicted to be in the Plin5 lipolytic barrier region, though this has not been tested. It is possible that $\mathrm{N}$ terminal lipid binding drives association of Plin5 with LDs, while our identified lipid binding pocket in the tether domain could be for FA transfer to mitochondria. However, whether the Plin5 C-terminal domain has lipid binding and transfer activity remains to be determined. The presence of acyl-CoA synthetases (ACSLs) at LD-organelle contact sites is an emerging theme. For example, ACSL1 on mitochondria interacts with LD-tethering proteins including SNAP23 (Young et al., 2018). Intriguingly, ACSL1 localization at mitochondria appears to be regulated by TANK-Binding Kinase 1 in response to fasting. Relocalization of ACSL1 to mitochondria subsequently increases $\beta$-oxidation (Huh et al., 2020). Similarly, the ER-LD tethering protein Snx14 interacts with ACSL4 (Datta et al., 2020), an isoenzyme of which localizes to ER/LDs (Küch et al., 2014). ACSL3 was identified as a protein at the ER membrane microdomains responsible for LD biogenesis, as well as on emerging and mature LDs (Kassan et al., 2013), suggesting that ACSL3 may also be present at ER-LD contact sites. The presence of ACSLs at LD-organelle contact sites is consistent with a prominent role of these membrane contact sites in channeling fatty acids to different metabolic fates.

What is the function of Plin5-mediated LD-mito contacts? Multiple functions have been proposed, based on seemingly contradictory results from mouse and cell models of Plin5 ablation or overexpression. Our results are consistent with previous work showing that Plin5 can switch between lipolytic barrier and pro-lipolytic activities, depending on its phosphorylation state (Pollak et al., 2015; Keenan et al., 2021). Our findings are also consistent with recently published work showing that expression of truncated Plin5 in human AC16 cardiomyocytes does not alter ß-oxidation relative to full-length Plin5 during acute starvation (Kien et al., 2022). Because that study did not use phosphomimetic Plin5 constructs, the authors concluded that 
the Plin5 mitochondrial tether domain is dispensable for FA oxidation. Here we have used a combination of phosphomimetic and truncation constructs to carefully tease apart the roles of the Plin5 lipolytic barrier and mitochondria tether domains. While the Plin5 lipolytic barrier domain can promote either FA storage or mobilization depending on its phosphorylation state, we found that the Plin5 mitochondrial tether domain was required for efficient flux of FAs from LDs to mitochondria, and their subsequent oxidation. Thus, we propose that a primary function of Plin5-mediated LD-mito contacts is to efficiently channel FAs from LDs to mitochondria. This occurs through interaction of the Plin5 C-terminal domain with Fatp4. Interestingly, LD-tomitochondria FA flux was completely ablated by knockdown of Fatp4, while LD-mito colocalization was reduced but not abolished by Fatp4 depletion (Fig. 5). This implies that additional mitochondrial proteins may interact with Plin5 at membrane contact sites, but that the Fatp4 interaction is specifically required for FA channeling. Indeed, we observed an interaction between the Plin5 lipolytic barrier domain and the mitofusin Mfn2 (Fig. 3). Mfn2 was previously reported to interact with Plin1, which shares a conserved N-terminal domain with Plin5 (Boutant et al., 2017). Thus, Plin5-mediated LD-mito contacts may have additional functions beyond FA channeling, including the regulation of mitochondrial fusion. Whether the interaction of Plin5 with binding partners including Fatp4 and Mfn2 occurs at the same or distinct membrane microdomains remains to be determined. The spatial relationship between Plin5/Fatp4 and other LD-mito contact proteins including SNAP23/ACSL1 (Jägerström et al., 2009; Young et al., 2018), MIGA2 (Freyre et al., 2019), and VPS13D/TSG101 (Wang et al., 2021) is also an area of future investigation.

The channeling of FAs directly from LDs to mitochondria at membrane contact sites during starvation has the benefit of preventing a build-up of cytoplasmic FAs, which can lead to mitochondrial toxicity (Nguyen et al., 2017). Alternative FA trafficking routes from LDs to mitochondria have previously been proposed. In hepatocytes, it was suggested that the primary route is through lipophagy of LDs, followed by fusion of lysosomes with the plasma membrane 
and subsequent reuptake of FAs and transport to mitochondria (Cui et al., 2020). Like FA transfer at LD-mito contact sites, lysosomal fusion with the plasma membrane would also prevent a buildup of cytoplasmic FAs, by releasing FAs extracellularly and allowing cytoplasmic FA concentration to be regulated at the reuptake step. Which mechanism is used in a given situation likely depends on the cell-type specific expression of regulatory proteins, as well as the stimulus initiating lipolysis/lipophagy.

In summary, we have identified the acyl-Co-A synthetase Fatp4 as a novel interactor of Plin5. We provide evidence that Plin5 interacts with Fatp4 at LD-mito contact sites to promote LD-to-mitochondria trafficking of FAs in a phosphorylation-dependent manner. This work helps to reconcile seemingly contradictory results seen in models of Plin5 ablation/overexpression by revealing the specific functions of the lipolytic barrier vs. mitochondrial tethering domains of Plin5.

\section{Acknowledgements}

We thank Amit Joshi and current members of the Cohen lab for helpful discussions and critical reading of the manuscript. We are grateful to Reginald Edwards for performing pilot LD tracking experiments. This research is based in part upon work conducted using the UNC Proteomics Core Facility, which is supported in part by P30 CA016086 Cancer Center Core Support Grant to the UNC Lineberger Comprehensive Cancer Center. Research reported in this publication was supported by the National Institute of General Medical Sciences of the National Institutes of Health under award numbers R35 GM133460 (S.C.) and F32 GM136027 (G.E.M.).

\section{Author Contributions}

G.E.M. and S.C. designed the research; G.E.M., C.M.S., W.E., and L.E.H. performed research; G.E.M., C.M.S., W.E., L.E.H., and E.L.K. analyzed data; L.E.H., E.L.K., and R.A.C. provided expertise and feedback, and G.E.M. and S.C. wrote the paper, with input from all coauthors. 


\section{Methods}

\section{Cell Culture and Transfection}

U2OS cells and C2C12 myobolasts were obtained from the UNC Tissue Culture Facility and maintained in Dulbecco's Modified Eagle Medium (DMEM) with 10\% FBS and $4 \mathrm{mM}$ glutamine (complete medium, CM). Cells were cultured on chambered cover glass (\#1.5 high performance cover glass, Cellvis), coated with $10 \mu \mathrm{g} / \mathrm{ml}$ fibronectin (MilliporeSigma). For starvation experiments, Hank's buffered salt solution (HBSS, 14025092) was purchased from Thermo Fisher. Cells were transfected with Mito-RFP, Mito-GFP, mOrange-SKL, or Plin5 constructs using Lipofectamine 2000 (Invitrogen) according to the manufacturer's instructions. RNA interference was performed using 25 nM ON-TARGETplus Mouse Fatp4 (J-063631-12-0002) siRNA or ON-TARGETplus non-targeting pool (Horizon Discovery) and DharmaFECT 1 (Horizon Discovery) according to the manufacturer's instructions.

\section{Plasmids}

Plasmids used are listed in Supplemental Table 2. Full-length Plin5, Plin5 truncations and Chimeric Plin5 or Fatp4 constructs were generated using HiFi DNA Assembly Master mix (New England Biolabs, E2621). Plin5 mutant constructs were generated using Q5® Site-Directed Mutagenesis Kit (New England Biolabs, E0554S) mEmerald-C1 and BFP-KDEL were used as vectors for mEmerald- and TagBFP- tagged constructs, respectively. For plasmid construction, all PCRs were performed using Q5 High Fidelity DNA polymerase (M0419; New England Biolabs) and restriction enzymes were from New England Biolabs. The following plasmids were kind gifts: pEYFP-C1-Plin5 from Carole Sztalryd (University of Maryland); TagBFP-KDEL from Gia Voeltz (University of Colorado); mEmerald-C1 and mOrange-SKL from Michael Davidson (Florida State University); Mito-GFP and Mito-RFP from Jennifer Lippincott-Schwartz (Janelia Research Campus).

\section{Inhibitors and Antibodies}

The following chemicals, dyes and antibodies were used: $10 \mu \mathrm{g} / \mathrm{ml}$ fibronectin (MilliporeSigma), $5 \mu \mathrm{M}$ BODIPY 558/568 C12 (Life Technologies), $5 \mathrm{nM}\left[1-{ }^{14} \mathrm{C}\right]$ oleate (Perkin Elmer), $50 \mathrm{ng} / \mathrm{ml}$ BODIPY 665/676 (Life Technologies), 100 nM MitoTracker Deep Red (Life Technologies), Lcarnitine (C0158-1G, Thermo Fisher), bovine serum albumin-fatty acid free (MilliporeSigma), anti-CPT1B (Abcam, ab104662), anti-GFP (Invitrogen, A10262), anti-Fatp4 (Abcam, ab200353), anti-Mfn2 (Cell Signaling, 11925), anti-BIII-tubulin (Abcam, ab18207), and anti-Plin5 (American Research Products, 03-GP31).

\section{Fluorescent FA Analog Pulse-Chase}

C2C12 cells were incubated with CM containing $5 \mu$ M BODIPY 558/568 C12 (Red C12, Life Technologies) for $16 \mathrm{hr}$. Cells were then washed three times with $\mathrm{CM}$, and chased for $1 \mathrm{hr}$ in order to allow the fluorescent lipids to incorporate into LDs. Cells were imaged immediately following pulse-chase labeling with Red C12. Cells were then washed three times with HBSS and imaged every $30 \mathrm{~min}$ for $4 \mathrm{hrs}$ in order to track the subcellular localization of FAs. Cells were transfected with Mito-GFP $24 \mathrm{hrs}$ prior to Red C12 labeling to visualize mitochondria. To label LDs, Bodipy 665/676 (Life Technologies) was added to cells at $50 \mathrm{ng} / \mathrm{ml} 16 \mathrm{hrs}$ prior to imaging and was present during imaging.

\section{Radioactive FA Pulse-Chase}

C2C12 cells were incubated with Krebs-Ringer bicarbonate HEPES buffer (KRBH) labeling buffer (KRBH containing $5.5 \mathrm{mM}$ glucose, $1 \mathrm{mM}$ carnitine, $0.25 \%$ FA free BSA) and $17 \mu \mathrm{M}$ [1${ }^{14} \mathrm{C}$ ]oleate $\left({ }^{14} \mathrm{C}\right.$-oleate, Perkin Elmer) for $16 \mathrm{hrs}$. Cells were then washed three times with $\mathrm{KRBH}$, and chased for $1 \mathrm{hr}$ in $\mathrm{KRBH}$ labeling buffer in order to allow ${ }^{14} \mathrm{C}$-oleate incorporation into 
cellular lipids. Following the pulse-chase cells were either collected to measure ${ }^{14} \mathrm{C}$-oleate incorporation, or incubated in HBSS to assess $\beta$-oxidation.

\section{Lipid Extraction and TLC}

Following the Radioactive FA pulse-chase, cells were washed twice with $\mathrm{KRBH}$ containing $1 \%$ FA Free BSA. Lipids were isolated by chloroform-methanol extraction. Following extraction lipids were dried by speedvac and resuspended in chloroform-methanol. Aliquoted samples were counted for total ${ }^{14} \mathrm{C}$-oleate incorporation. Remaining samples were spotted onto silica gel preparative TLC plates (MilliporeSigma). Separation of lipids was performed by developing the plates in a 2-step solvent system of chloroform:methanol:ammonium hydroxide, 65:25:4 (vol/vol) followed by heptane:isopropyl ether:acetic acid (15:10:1) (vol/vol). Radiolabeled were visualized using a Bioscan AR-2000 radio-TLC imaging scanner (Bioscan).

\section{ASM Measurement}

Following Radioactive FA pulse-chase, cells were washed three times with HBSS and incubated with HBSS containing $1 \mathrm{mM}$ carnitine. $500 \mu \mathrm{l}$ of media was collected after $6 \mathrm{hrs}$ and incubated with $12.5 \mu \mathrm{l}$ of $25 \%$ FA-free BSA overnight at $4{ }^{\circ} \mathrm{C}$ followed by centrifugation at 20,800 gfor 20 min. Supernatants were washed once more with $12.5 \mu \mathrm{l}$ of $25 \%$ FA-free BSA and spun $20,800 \mathrm{~g}$ for $20 \mathrm{~min}$, before aliquots of the supernatant were counted for ${ }^{14} \mathrm{C}$-labeled ASM.

\section{Isolation of GFP-Plin5 Complexes for Mass Spectrometry}

$\mathrm{mEmerald}$ protein complex isolation for mass spectrometry was performed as previously described(Kaltenbrun et al., 2013)utilizing GFP-Trap magnetic beads (Chromotek). Briefly, U2OS cells expressing mEmerald constructs were washed twice with cold PBS (Thermo Fisher, $\# 14190144$ ), harvested by scraping with a cell lifter, and centrifuged at $350 \mathrm{~g}$ for $10 \mathrm{~min}$ at $4^{\circ} \mathrm{C}$. Cell pellets were resuspended in $20 \mathrm{~mL}$ resuspension buffer $(20 \mathrm{mM}$ HEPES, pH 7.4, 1.2\% polyvinylpyrrolidone) with protease (MiliporeSigma, \#8340) and phosphatase inhibitors (MiliporeSigma, \#5726 and \#P0044), and snap frozen in liquid nitrogen. Cells were lysed by cryogenic grinding using a MM 301 Mixer Mill (10 cycles, $2.5 \mathrm{~min}$ at $30 \mathrm{~Hz}$ ) (Retsch). Lysate was resuspended in MS lysis buffer (20mM K-HEPES pH 7.4, 150mM NaCl, 100mM KOAc, $2 \mathrm{mM}$ $\mathrm{MgCl} 2,0.1 \%$ Tween-20, $1 \mu \mathrm{m} \mathrm{ZnCl} 21 \mu \mathrm{mCaCl} 2,0.5 \%$ Triton X-100) with protease and phosphatase inhibitors ( $5 \mathrm{ml} / \mathrm{g}$ cells). Resuspended lysate was homogenized using a Polytron (Kinematica) $(2 \times 15 \mathrm{sec}$ at $25,000 \mathrm{RPM})$ and pelleted at $2500 \mathrm{~g}$ at $4^{\circ} \mathrm{C}$. Cleared lysate was incubated with $50 \mu$ l equilibrated GFP-Trap magnetic beads by rotating for $1 \mathrm{hr}$ at $4^{\circ} \mathrm{C}$. Beads were washed 6 times with $1 \mathrm{ml}$ of lysis buffer. GFP complexes were eluted from beads in $40 \mu \mathrm{l}$ 1X LDS Sample Buffer (Invitrogen) at $70^{\circ} \mathrm{C}$ for $15 \mathrm{~min}$. Eluted complexes were alkylated with $100 \mathrm{mM}$ iodoacetamide for $1 \mathrm{hr}$ at room temperature prior to mass spectrometry analysis.

\section{Liquid Chromatography- Tandem Mass Spectrometry (LC-MS/MS) Analysis}

Immunoprecipitated samples were subjected to SDS-PAGE and stained with coomassie. Lanes $(1 \mathrm{~cm})$ for each sample were excised and the proteins were reduced with $5 \mathrm{mM}$ DTT for 30 min at $55 \mathrm{C}$, alkylated with $15 \mathrm{mM}$ IAA for $45 \mathrm{~min}$ in the dark at room temperature, and in-gel digested with trypsin overnight at $37^{\circ} \mathrm{C}$. Peptides were extracted, desalted with $\mathrm{C} 18$ spin columns (Pierce) and dried via vacuum centrifugation. Peptide samples were stored at $-80^{\circ} \mathrm{C}$ until further analysis.

Each sample was analyzed in duplicate by LC-MS/MS using an Easy nLC 1200 coupled to a QExactive HF (Thermo Scientific). Samples were injected onto an Easy Spray PepMap C18 column $(75 \mu \mathrm{m}$ id $\times 25 \mathrm{~cm}, 2 \mu \mathrm{m}$ particle size) (Thermo Scientific) and separated over a $60 \mathrm{~min}$ method. The gradient for separation consisted of $5-45 \%$ mobile phase B at a $250 \mathrm{nl} / \mathrm{min}$ flow rate, where mobile phase $A$ was $0.1 \%$ formic acid in water and mobile phase $B$ consisted of 
$0.1 \%$ formic acid in $80 \%$ ACN. The QExactive HF was operated in data-dependent mode where the 15 most intense precursors were selected for subsequent HCD fragmentation. Resolution for the precursor scan (m/z 350-1600) was set to 60,000, while MS/MS scans resolution was set to 15,000 . The normalized collision energy was set to $27 \%$ for HCD. Peptide match was set to preferred, and precursors with unknown charge or a charge state of 1 and $\geq 7$ were excluded.

Raw data files were processed using Proteome Discoverer version 2.1 (Thermo Scientific). Peak lists were searched against a reviewed Uniprot human database (containing 20,414 protein sequences, downloaded January 2019). appended with a common contaminants database. The following parameters were used to identify tryptic peptides for protein identification: 10 ppm precursor ion mass tolerance; 0.02 Da product ion mass tolerance; up to two missed trypsin cleavage sites; carbamidomethylation of Cys was set as a fixed modification; oxidation of was set as a variable modification. Scaffold (version 4.7.3, Proteome Software) was used to validate MS/MS based peptide and protein identifications, and to provide relative quantitation. Peptide identifications were accepted if they could be established at greater than $95 \%$ probability to achieve an FDR less than $0.1 \%$ by the Scaffold Local FDR algorithm. Protein identifications were accepted if they could be established at greater than $99.0 \%$ probability and contained at least 2 identified peptides. SAINT (Significance Analysis of INTeractome) (Choi et al., 2012) was used to identify Plin5 interactors. Proteins were identified as Plin5 interactors if they had a FC_A (Average enrichment relative to Em control) score greater than 2.0 and a SAINT score greater than 0.75 . Relative quantitation was performed using the calculated quantitative values (normalized peak area) within Scaffold.

Isolation of GFP-Plin5 Complexes for Co-Immunoprecipitation/Western Blot Isolation of mEmerald protein complexes for co-immunoprecipitation was performed utilizing the same harvesting method used for mass spectrometry. Following cell harvesting, Coimmunoprecipitation was performed utilizing GFP-Trap magnetic beads (Chromotek) following manufacturer protocols with slight modification. Cell pellets were resuspended in $400 \mu \mathrm{L}$ of CoIP Lysis Buffer (10 mM Tris/Cl pH 7.5; $150 \mathrm{mM} \mathrm{NaCl;} 0.5 \mathrm{mM}$ EDTA; 0.5\% NP-40) with protease and phosphatase inhibitors. Cell suspension was incubated on ice for 30 min with pipetting every 10 minutes and pelleted at $20,000 \mathrm{~g}$ for $10 \mathrm{~min}$ at $4{ }^{\circ} \mathrm{C} .600 \mu \mathrm{l}$ of Co-IP Dilution Buffer (10 $\mathrm{mM}$ Tris- $\mathrm{Cl} \mathrm{pH} \mathrm{7.5;} 150 \mathrm{mM} \mathrm{NaCl} ; 0.5 \mathrm{mM}$ EDTA) was added to cleared lysate. Diluted lysate was incubated with $25 \mu$ lequilibrated GFP-Trap magnetic beads by rotating for $1 \mathrm{hr}$ at $4{ }^{\circ} \mathrm{C}$. Beads were washed 3 times with 500 ul of Co-IP Dilution Buffer. GFP complexes were eluted from beads in $30 \mu \mathrm{l} 6 \mathrm{X}$ Laemmli Sample Buffer at $95^{\circ} \mathrm{C}$ for $10 \mathrm{~min}$. Protein complexes were examined by Western blotting.

\section{Microscopy, Image Processing, Analysis, and Statistics}

Images were acquired on an inverted Zeiss 800/Airyscan laser scanning confocal microscope equipped with 405, 488, 561 and 647 nm diode lasers, and Galium Arsenide Phosphid (GaAsP) and Airyscan detectors. Confocal and Airyscan images were acquired using a 63x/1.4 NA objective lens, at $37{ }^{\circ} \mathrm{C}$ and $5 \% \mathrm{CO}_{2}$ (Carl Zeiss, Oberkochen, Germany). For movies S1-S4, Airyscan timelapse images were acquired every $27 \mathrm{~s}$ for 10 frames. Airyscan images were processed in Zen software (Carl Zeiss) using a processing strength of 6.0.

Image brightness and contrast were adjusted in Adobe Photoshop CS. Images were analyzed using Image $(\mathrm{NIH})$.

For organelle colocalization, masks of each organelle were created using the corresponding channels. For overlap of LDs with organelles (mitochondria, peroxisomes), the percentage of LD pixels colocalized with the organelle versus total LD pixels was calculated. For overlap of 
peroxisomes and mitochondria the percentage of peroxisome pixels colocalized with the organelle versus total peroxisome pixels was calculated.

LDs were tracked using the TrackMate (Tinevez et al., 2017) plugin, an ImageJ implementation of the linear assignment problem (LAP) tracking algorithm (Jaqaman et al., 2008).

For fluorescent FA pulse-chase assays, images were analyzed using ImageJ to determine total fluorescence intensity. To quantify the fluorescence intensity of Red $\mathrm{C} 12$ in mitochondria and LDs, we made mitochondria and LD masks using the Mito-GFP and Bodipy 665/667 channels respectively, and then the fluorescence intensity in the Red $\mathrm{C} 12$ channel was calculated across the entire mask.

Data were expressed as means, error bars represent \pm SEM. Statistical analysis among groups was performed using Student's t test.

\section{References}

Benador, I. Y. et al. (2018) 'Mitochondria Bound to Lipid Droplets Have Unique Bioenergetics, Composition, and Dynamics that Support Lipid Droplet Expansion', Cell Metabolism. Cell Press, 27(4), pp. 869-885.e6. Available at:

https://www.sciencedirect.com/science/article/pii/S1550413118301815?via\%3Dihub (Accessed: 5 September 2018).

Benador, I. Y. et al. (2019) 'Mitochondria Bound to Lipid Droplets: Where Mitochondrial Dynamics Regulate Lipid Storage and Utilization', Cell Metabolism. Cell Press, 29(4), pp. 827835. doi: 10.1016/J.CMET.2019.02.011.

Bohnert, M. (2020) 'Tether Me, Tether Me Not-Dynamic Organelle Contact Sites in Metabolic Rewiring', Developmental Cell. Cell Press, 54(2), pp. 212-225. doi:

10.1016/J.DEVCEL.2020.06.026.

Bosma, M. et al. (2013) 'Overexpression of PLIN5 in skeletal muscle promotes oxidative gene expression and intramyocellular lipid content without compromising insulin sensitivity', Biochimica et Biophysica Acta (BBA) - Molecular and Cell Biology of Lipids. Elsevier, 1831(4), pp. 844-852. doi: 10.1016/J.BBALIP.2013.01.007.

Boutant, M. et al. (2017) 'Mfn2 is critical for brown adipose tissue thermogenic function', The EMBO Journal. John Wiley \& Sons, Ltd, 36(11), pp. 1543-1558. doi:

10.15252/EMBJ.201694914.

Cho, K. F. et al. (2020) 'Split-TurbolD enables contact-dependent proximity labeling in cells', Proceedings of the National Academy of Sciences. National Academy of Sciences, 117(22), pp. 12143-12154. doi: 10.1073/PNAS.1919528117.

Choi, H. et al. (2012) 'Analyzing protein-protein interactions from affinity purification-mass spectrometry data with SAINT', Current protocols in bioinformatics / editoral board, Andreas D. Baxevanis ... [et al.]. NIH Public Access, CHAPTER(SUPPL.39), p. Unit8.15. doi: 10.1002/0471250953.BI0815S39.

Cui, W. et al. (2020) 'Lipophagy-derived fatty acids undergo extracellular efflux via lysosomal exocytosis', https://doi.org/10.1080/15548627.2020.1728097. Taylor \& Francis, 17(3), pp. 690705. doi: 10.1080/15548627.2020.1728097.

Daemen, S., Van Polanen, N. and Hesselink, M. K. C. (2018) 'The effect of diet and exercise on 
lipid droplet dynamics in human muscle tissue', The Journal of experimental biology. J Exp Biol, 221(Pt Suppl 1). doi: 10.1242/JEB.167015.

Datta, S. et al. (2020) 'Snx14 proximity labeling reveals a role in saturated fatty acid metabolism and ER homeostasis defective in SCAR20 disease', Proceedings of the National Academy of Sciences of the United States of America. National Academy of Sciences, 117(52), pp. 3328233294. doi: 10.1073/PNAS.2011124117/-/DCSUPPLEMENTAL.

Devries, M. C. et al. (2013) 'Endurance Training Modulates Intramyocellular Lipid Compartmentalization and Morphology in Skeletal Muscle of Lean and Obese Women', The Journal of Clinical Endocrinology \& Metabolism. Oxford Academic, 98(12), pp. 4852-4862. doi: 10.1210/JC.2013-2044.

Drevinge, C. et al. (2016) 'Perilipin 5 is protective in the ischemic heart', International Journal of Cardiology. Elsevier, 219, pp. 446-454. doi: 10.1016/J.IJCARD.2016.06.037.

Freyre, C. A. C. et al. (2019) 'MIGA2 Links Mitochondria, the ER, and Lipid Droplets and Promotes De Novo Lipogenesis in Adipocytes', Molecular Cell. Cell Press, 76(5), pp. 811825.e14. doi: 10.1016/J.MOLCEL.2019.09.011.

Gallardo-Montejano, V. I. et al. (2016) 'Nuclear Perilipin 5 integrates lipid droplet lipolysis with PGC-1a/SIRT1-dependent transcriptional regulation of mitochondrial function', Nature Communications. Nature Publishing Group, 7(1), p. 12723. doi: 10.1038/ncomms12723.

Gallardo-Montejano, V. I. et al. (2021) 'Perilipin 5 links mitochondrial uncoupled respiration in brown fat to healthy white fat remodeling and systemic glucose tolerance', Nature Communications 2021 12:1. Nature Publishing Group, 12(1), pp. 1-18. doi: 10.1038/s41467021-23601-2.

Gemmink, A. et al. (2018) 'Super-resolution microscopy localizes perilipin 5 at lipid dropletmitochondria interaction sites and at lipid droplets juxtaposing to perilipin 2', Biochimica et Biophysica Acta (BBA) - Molecular and Cell Biology of Lipids. Elsevier, 1863(11), pp. 14231432. doi: 10.1016/J.BBALIP.2018.08.016.

Hall, A. M. et al. (2005) 'Enzymatic Properties of Purified Murine Fatty Acid Transport Protein 4 and Analysis of Acyl-CoA Synthetase Activities in Tissues from FATP4 Null Mice *', Journal of Biological Chemistry. Elsevier, 280(12), pp. 11948-11954. doi: 10.1074/JBC.M412629200.

Herms, A. et al. (2015) 'AMPK activation promotes lipid droplet dispersion on detyrosinated microtubules to increase mitochondrial fatty acid oxidation', Nature Communications 2015 6:1. Nature Publishing Group, 6(1), pp. 1-14. doi: 10.1038/ncomms8176.

Herrmann, T. et al. (2001) 'Mouse fatty acid transport protein 4 (FATP4): Characterization of the gene and functional assessment as a very long chain acyl-CoA synthetase', Gene. Elsevier, 270(1-2), pp. 31-40. doi: 10.1016/S0378-1119(01)00489-9.

Huh, J. Y. et al. (2020) 'TANK-Binding Kinase 1 Regulates the Localization of Acyl-CoA Synthetase ACSL1 to Control Hepatic Fatty Acid Oxidation', Cell Metabolism. Cell Press, 32(6), pp. 1012-1027.e7. doi: 10.1016/J.CMET.2020.10.010.

Jägerström, S. et al. (2009) 'Lipid droplets interact with mitochondria using SNAP23', Cell Biology International. John Wiley \& Sons, Ltd, 33(9), pp. 934-940. doi:

10.1016/J.CELLBI.2009.06.011.

Jaqaman, K. et al. (2008) 'Robust single-particle tracking in live-cell time-lapse sequences', Nature methods. Nat Methods, 5(8), pp. 695-702. doi: 10.1038/NMETH.1237.

Jeppesen, J. et al. (2012) 'Enhanced Fatty Acid Oxidation and FATP4 Protein Expression after 
Endurance Exercise Training in Human Skeletal Muscle', PLOS ONE. Public Library of Science, 7(1), p. e29391. doi: 10.1371/JOURNAL.PONE.0029391.

Jia, Z., Moulson, C. L., et al. (2007) 'Fatty Acid Transport Protein 4 Is the Principal Very Long Chain Fatty Acyl-CoA Synthetase in Skin Fibroblasts', Journal of Biological Chemistry. Elsevier, 282(28), pp. 20573-20583. doi: 10.1074/JBC.M700568200.

Jia, Z., Pei, Z., et al. (2007) 'The fatty acid transport protein (FATP) family: Very long chain acylCoA synthetases or solute carriers?', Journal of Molecular Neuroscience. Springer, 33(1), pp. 25-31. doi: 10.1007/S12031-007-0038-Z/FIGURES/6.

Kaltenbrun, E. et al. (2013) 'A Gro/TLE-NuRD corepressor complex facilitates Tbx20-dependent transcriptional repression', Journal of proteome research. J Proteome Res, 12(12), pp. 53955409. doi: 10.1021/PR400818C.

Kannan, M. et al. (2017) 'Phosphatidylserine synthesis at membrane contact sites promotes its transport out of the ER', Journal of Lipid Research. Elsevier, 58(3), pp. 553-562. doi: 10.1194/JLR.M072959.

Kassan, A. et al. (2013) 'Acyl-CoA synthetase 3 promotes lipid droplet biogenesis in ER microdomains', Journal of Cell Biology. The Rockefeller University Press, 203(6), pp. 985-1001. doi: 10.1083/JCB.201305142/VIDEO-5.

Keenan, S. N. et al. (2021) 'Perilipin 5 S155 phosphorylation by PKA is required for the control of hepatic lipid metabolism and glycemic control', Journal of Lipid Research. American Society for Biochemistry and Molecular Biology, 62. doi: 10.1194/JLR.RA120001126.

Kien, B. et al. (2022) 'Lipid droplet-mitochondria coupling via Perilipin 5 augments respiratory capacity but is dispensable for FA oxidation', Journal of Lipid Research. Elsevier, p. 100172. doi: 10.1016/J.JLR.2022.100172.

Kilwein, M. D. and Welte, M. A. (2019) 'Lipid Droplet Motility and Organelle Contacts', Contact. SAGE Publications, 2(585), p. 251525641989568. doi: 10.1177/2515256419895688.

Kimmel, A. R. and Sztalryd, C. (2016) 'The Perilipins: Major Cytosolic Lipid Droplet-Associated Proteins and Their Roles in Cellular Lipid Storage, Mobilization, and Systemic Homeostasis*', http://dx.doi.org/10.1146/annurev-nutr-071813-105410. Annual Reviews , 36, pp. 471-509. doi: 10.1146/ANNUREV-NUTR-071813-105410.

Küch, E. M. et al. (2014) 'Differentially localized acyl-CoA synthetase 4 isoenzymes mediate the metabolic channeling of fatty acids towards phosphatidylinositol', Biochimica et Biophysica Acta (BBA) - Molecular and Cell Biology of Lipids. Elsevier, 1841(2), pp. 227-239. doi: 10.1016/J.BBALIP.2013.10.018.

Kuramoto, K. et al. (2012) 'Perilipin 5, a Lipid Droplet-binding Protein, Protects Heart from Oxidative Burden by Sequestering Fatty Acid from Excessive Oxidation', Journal of Biological Chemistry. Elsevier, 287(28), pp. 23852-23863. doi: 10.1074/JBC.M111.328708.

Lobo, S. et al. (2007) 'Fatty acid metabolism in adipocytes: functional analysis of fatty acid transport proteins 1 and 4', Journal of Lipid Research. Elsevier, 48(3), pp. 609-620. doi: 10.1194/JLR.M600441-JLR200.

MacPherson, R. E. K. et al. (2013) 'Skeletal muscle PLIN proteins, ATGL and CGI-58, interactions at rest and following stimulated contraction', American Journal of Physiology Regulatory, Integrative and Comparative Physiology. American Physiological Society, 304(8), p. R644. doi: 10.1152/AJPREGU.00418.2012.

Mason, R. R. et al. (2014) 'PLIN5 deletion remodels intracellular lipid composition and causes 
insulin resistance in muscle', Molecular Metabolism. Elsevier, 3(6), pp. 652-663. doi: 10.1016/J.MOLMET.2014.06.002.

Montgomery, M. K. et al. (2019) 'Perilipin 5 Deletion in Hepatocytes Remodels Lipid Metabolism and Causes Hepatic Insulin Resistance in Mice', Diabetes. American Diabetes Association, 68(3), pp. 543-555. doi: 10.2337/DB18-0670.

Najt, C. P. et al. (2020) 'Lipid Droplet-Derived Monounsaturated Fatty Acids Traffic via PLIN5 to Allosterically Activate SIRT1', Molecular Cell. Cell Press, 77(4), pp. 810-824.e8. doi: 10.1016/J.MOLCEL.2019.12.003.

Nguyen, T. B. et al. (2017) 'DGAT1-dependent lipid droplet biogenesis protects mitochondrialfunction during starvation-induced autophagy', Developmental cell. NIH Public Access, 42(1), p. 9. doi: 10.1016/J.DEVCEL.2017.06.003.

Olzmann, J. A. and Carvalho, P. (2018) 'Dynamics and functions of lipid droplets', Nature Reviews Molecular Cell Biology 2018 20:3. Nature Publishing Group, 20(3), pp. 137-155. doi: 10.1038/s41580-018-0085-z.

Pollak, N. M. et al. (2015) 'The interplay of protein kinase A and perilipin 5 regulates cardiac lipolysis.', The Journal of biological chemistry. American Society for Biochemistry and Molecular Biology, 290(3), pp. 1295-306. doi: 10.1074/jbc.M114.604744.

Prinz, W. A. (2014) 'Bridging the gap: membrane contact sites in signaling, metabolism, and organelle dynamics.', The Journal of cell biology. Rockefeller University Press, 205(6), pp. 75969. doi: 10.1083/jcb.201401126.

Prinz, W. A., Toulmay, A. and Balla, T. (2019) 'The functional universe of membrane contact sites', Nature Reviews Molecular Cell Biology 2019 21:1. Nature Publishing Group, 21(1), pp. 724. doi: 10.1038/s41580-019-0180-9.

Rambold, A. S., Cohen, S. and Lippincott-Schwartz, J. (2015) 'Fatty Acid Trafficking in Starved Cells: Regulation by Lipid Droplet Lipolysis, Autophagy, and Mitochondrial Fusion Dynamics', Developmental Cell. Cell Press, 32(6), pp. 678-692. doi: 10.1016/J.DEVCEL.2015.01.029.

Roy, A., Kucukural, A. and Zhang, Y. (2010) 'I-TASSER: a unified platform for automated protein structure and function prediction', Nature Protocols 2010 5:4. Nature Publishing Group, 5(4), pp. 725-738. doi: 10.1038/nprot.2010.5.

Schuldiner, M. and Bohnert, M. (2017) 'A different kind of love - lipid droplet contact sites', Biochimica et Biophysica Acta (BBA) - Molecular and Cell Biology of Lipids. Elsevier, 1862(10), pp. 1188-1196. doi: 10.1016/J.BBALIP.2017.06.005.

Shepherd, S. O. et al. (2013) 'Sprint interval and traditional endurance training increase net intramuscular triglyceride breakdown and expression of perilipin 2 and 5', The Journal of Physiology. Wiley-Blackwell, 591(Pt 3), p. 657. doi: 10.1113/JPHYSIOL.2012.240952.

Steensels, S. et al. (2020) 'Acyl-Coenzyme A Thioesterase 9 Traffics Mitochondrial Short-Chain Fatty Acids Toward De Novo Lipogenesis and Glucose Production in the Liver', Hepatology. John Wiley \& Sons, Ltd, 72(3), pp. 857-872. doi: 10.1002/HEP.31409.

Tarnopolsky, M. A. et al. (2007) 'Influence of endurance exercise training and sex on intramyocellular lipid and mitochondrial ultrastructure, substrate use, and mitochondrial enzyme activity', American Journal of Physiology - Regulatory Integrative and Comparative Physiology. American Physiological Society, 292(3), pp. 1271-1278. doi: 10.1152/AJPREGU.00472.2006/ASSET/IMAGES/LARGE/ZH60030756800003.JPEG.

Tinevez, J. Y. et al. (2017) 'TrackMate: An open and extensible platform for single-particle 
tracking', Methods. Academic Press, 115, pp. 80-90. doi: 10.1016/J.YMETH.2016.09.016. Valm, A. M. et al. (2017) 'Applying systems-level spectral imaging and analysis to reveal the organelle interactome.', Nature. Howard Hughes Medical Institute, 546(7656), pp. 162-167. doi: 10.1038/nature22369.

Wang, H., Sreenivasan, U., et al. (2011) 'Perilipin 5, a lipid droplet-associated protein, provides physical and metabolic linkage to mitochondria', Journal of Lipid Research. American Society for Biochemistry and Molecular Biology, 52(12), p. 2159. doi: 10.1194/JLR.M017939.

Wang, H., Bell, M., et al. (2011) 'Unique Regulation of Adipose Triglyceride Lipase (ATGL) by Perilipin 5, a Lipid Droplet-associated Protein', The Journal of Biological Chemistry. American Society for Biochemistry and Molecular Biology, 286(18), p. 15707. doi: 10.1074/JBC.M110.207779.

Wang, H. et al. (2013) 'Cardiomyocyte-specific perilipin 5 overexpression leads to myocardial steatosis and modest cardiac dysfunction', Journal of Lipid Research. Elsevier, 54(4), pp. 953965. doi: 10.1194/JLR.M032466.

Wang, H. et al. (2016) 'Seipin is required for converting nascent to mature lipid droplets', eLife. eLife Sciences Publications Ltd, 5(AUGUST). doi: 10.7554/ELIFE.16582.

Wang, H. and Sztalryd, C. (2011) 'Oxidative tissue: perilipin 5 links storage with the furnace.', Trends in endocrinology and metabolism: TEM. Elsevier, 22(6), pp. 197-203. doi: 10.1016/j.tem.2011.03.008.

Wang, J. et al. (2021) 'An ESCRT-dependent step in fatty acid transfer from lipid droplets to mitochondria through VPS13D-TSG101 interactions', Nature Communications 2021 12:1. Nature Publishing Group, 12(1), pp. 1-16. doi: 10.1038/s41467-021-21525-5.

$\mathrm{Xu}, \mathrm{D}$. and Zhang, Y. (2012) 'Ab initio protein structure assembly using continuous structure fragments and optimized knowledge-based force field', Proteins: Structure, Function, and Bioinformatics. John Wiley \& Sons, Ltd, 80(7), pp. 1715-1735. doi: 10.1002/PROT.24065.

$\mathrm{Xu}, \mathrm{D}$. and Zhang, Y. (2013) 'Toward optimal fragment generations for ab initio protein structure assembly', Proteins: Structure, Function, and Bioinformatics. John Wiley \& Sons, Ltd, 81(2), pp. 229-239. doi: 10.1002/PROT.24179.

Yang, J. et al. (2015) 'The I-TASSER Suite: protein structure and function prediction', Nature Methods. NIH Public Access, 12(1), pp. 7-8. doi: 10.1038/nmeth.3213.

Yang, J. and Zhang, Y. (2015) 'I-TASSER server: new development for protein structure and function predictions', Nucleic Acids Research. Oxford University Press, 43(Web Server issue), p. W174. doi: 10.1093/NAR/GKV342.

Young, P. A. et al. (2018) 'Long-chain acyl-CoA synthetase 1 interacts with key proteins that activate and direct fatty acids into niche hepatic pathways', Journal of Biological Chemistry. Elsevier, 293(43), pp. 16724-16740. doi: 10.1074/JBC.RA118.004049.

Zechner, R., Madeo, F. and Kratky, D. (2017) 'Cytosolic lipolysis and lipophagy: two sides of the same coin', Nature Reviews Molecular Cell Biology 2017 18:11. Nature Publishing Group, 18(11), pp. 671-684. doi: 10.1038/nrm.2017.76.

Zheng, P. et al. (2017) 'Plin5 alleviates myocardial ischaemia/reperfusion injury by reducing oxidative stress through inhibiting the lipolysis of lipid droplets'. Nature Publishing Group, 7(1). doi: $10.1038 /$ srep42574. 


\section{Figure Legends}

Figure 1. Plin5 contains a C-terminal domain sufficient to promote LD-mito contacts (A-B) Structure predictions of Plin5. (A) I-TASSER structure prediction for Plin5 showing the conserved perilipin lipolytic barrier domain (orange) and a putative C-terminal tether domain with no strong structural prediction (purple). (B) QUARK de-novo structure prediction for the putative tether domain shows a two alpha-helix structure containing a hydrophobic pocket.

(C) Schematic of mEmerald-tagged full-length Plin5 and C-terminal truncations.

(D) Micrographs of U2OS cells transfected with Mito-RFP and the indicated Em-Plin5 construct, and labeled for LDs with Bodipy 665/676. Merged micrograph includes Mito-RFP and Bodipy $665 / 667$ channels to demonstrate co-localization (white).

(E-F) Quantification of images from (D). (E) Colocalization of LDs with mitochondria was measured as \% LD pixels overlapping with mitochondria, and (F) LD area/cell was measured, normalized to control.

(G-H) Design of constructs to target the C-terminal region of Plin5 to the LD surface using the hairpin (HP) domain of Gpat4. (G) Cartoon depiction of constructs used to target Plin5 Cterminal region to LD surface. Cartoon created with Biorender.com. $(\mathrm{H})$ Schematic of mEmerald-tagged HP-Plin5 constructs.

(I) Micrographs of U2OS cells transfected with Mito-RFP and the indicated Em-Plin5 construct, and labeled for LDs with Bodipy 665/676. Merged micrograph includes Mito-RFP and Bodipy $665 / 667$ channels to demonstrate co-localization (white).

$(\mathrm{J}-\mathrm{K})$ Quantification of images from $(\mathrm{I})$. (J) Colocalization of LDs with mitochondria was measured as \% LD pixels overlapping with mitochondria, and (K) LD area/cell was measured, normalized to control.

Scale bar, $10 \mu \mathrm{m}$. Data are expressed as means, error bars represent \pm SEM. ${ }^{* *} p<0.01,{ }^{* *} p<$ 0.001 .

Figure 2. Plin5 phosphorylation and LD-mito contacts drive ß-oxidation by increasing LDto-mitochondria FA trafficking

(A) Schematic representation of FA analog pulse-chase assay design: C2C12 myoblasts were pulsed with FA analog overnight to allow accumulation into LDs, washed, and chased with complete media (CM) for $1 \mathrm{hr}$. Following chase, incorporation of FA analog into LDs was measured. Cells were then incubated in HBSS for 4 or 6 hrs and FA subcellular localization or oxidation was measured.

(B-D) Fluorescent pulse-chase assay. Control C2C12 myoblasts or C2C12 myoblasts overexpressing indicated Plin5 constructs were pulsed with Red C12 using the assay described in (A) to monitor FA trafficking from LDs to mitochondria. Following CM chase, cells were imaged to measure incorporation of Red C12 into LDs. Cells were then incubated in HBSS and imaged every 30 min for 4 hrs to monitor FA localization. (B) Representative images of control cells following CM chase or $4 \mathrm{hr}$ HBSS incubation. (C) Red C12 incorporation into LDs following $\mathrm{CM}$ chase was measured, normalized to control cells. (D) Red C12 accumulation into mitochondria relative to initial LD incorporation following HBSS starvation was measured, normalized to control cells.

(E-F) Radioactive pulse-chase assay. Control C2C12 myoblasts or C2C12 myoblasts overexpressing indicated Em-Plin5 constructs were pulsed with a radioactive FA analog [1${ }^{14} \mathrm{C}$ ]oleate using the assay described in $(\mathrm{A})$ to monitor FA $\beta$-oxidation. Following media chase cells were either collected for TLC to measure incorporation of $\left[1-{ }^{14} \mathrm{C}\right]$ oleate into cellular lipids or incubated in HBSS for 6 hrs to measure $\beta$-oxidation. (E) $\left[1-{ }^{14} \mathrm{C}\right]$ oleate incorporation into lipid species following CM chase was measure by TLC, normalized to control cells. Lipid species abbreviations: Cholesterol Ester (CE), Triacylglycerol (TAG), Diacylglycerol (DAG), Free Fatty Acid (FFA), Phosphatidylcholine (PC), Phosphatidylethanolamine (PE), and Phosphatidylserine 
(PS). (F) $\beta$-oxidation following $6 \mathrm{hr}$ incubation in HBSS was assessed by measuring ASM released into media relative to $\left[1-{ }^{14} \mathrm{C}\right]$ oleate incorporation, normalized to control cells. Scale bar, $10 \mu \mathrm{m}$. Data are expressed as means, error bars represent \pm SEM. ${ }^{*} p<0.05,{ }^{* *} p<$ $0.01,{ }^{* * *} p<0.001^{* * * *} p<0.0001$.

Figure 3. The Plin5 tether domain interacts with acyl-CoA synthetase Fatp4 (A-D) Plin5 protein complexes were affinity purified from U2OS cells transfected with the indicated Em-Plin5 construct and analyzed by mass spectrometry. (A) Graphical representation of Plin5 associated protein complexes which show decreased abundance in Plin5C $\Delta$ affinity purifications. Circle size and color represent relative enrichment, border color represents statistical significance. (B) Calculated fold change for each Plin5 associated protein and proposed function. Proteins highlighted in grey show mitochondrial subcellular localization. (C) Western blot and (D) quantification of Mfn2 and Fatp4 abundance in affinity purified Plin5 protein complexes. Protein abundance is relative to Plin5, normalized to WT-Plin5 affinity purification.

Data are expressed as means, error bars represent \pm SEM. ${ }^{*} p<0.05,{ }^{* *} p<0.01,{ }^{* * *} p<0.001$.

\section{Figure 4. Plin5 and Fatp4 drive ectopic membrane contact sites}

(A-B) Design of constructs targeting the tether domain of Plin5 to the cytosolic face of the peroxisome membrane using the membrane targeting sequence of Pex3 (Pex). (A) Schematic of mEmerald-tagged Pex constructs. (B) Cartoon depiction of construct used to target Plin5 tether domain to peroxisome membrane. Cartoon created with Biorender.com.

(C) Micrographs of U2OS cells transfected with mOrange-SKL and indicated Pex construct, mitochondria labeled with MitoTracker Deep Red FM. Merged micrograph includes mOrangeSKL and MitoTracker Deep Red FM channels to demonstrate co-localization (white).

(D) Quantification of images from (C). Colocalization of peroxisomes with mitochondria was measured as \% peroxisome pixels overlapping with mitochondria.

(E-F) Design of constructs targeting the cytosolic domain of Fatp4 to the cytosolic face of the peroxisome membrane using Pex. (E) Schematic of TagBFP-tagged Pex constructs. (F) Cartoon depiction of construct used to target Fatp4 cytoplasmic domain to peroxisome membrane.

(G) Micrographs of U2OS cells transfected with mOrange-SKL and indicated HP/Pex targeted constructs, LDs labeled with Bodipy 665/676. Merged micrograph includes mOrange-SKL and Bodipy 665/676 channels to demonstrate co-localization (white).

(H) Quantification of images from G. Colocalization of LDs with peroxisomes was measured as $\%$ LD pixels overlapping with peroxisomes.

Scale bar, $10 \mu \mathrm{m}$. Data are expressed as means, error bars represent \pm SEM. ${ }^{* *} p<0.01$.

Figure 5. Loss of Fatp4 impairs Plin5-induced LD-mito contacts and FA trafficking (A) Micrographs of C2C12 myoblasts transfected for $24 \mathrm{hr}$ with non-targeting or Fatp4 siRNA. Following RNAi, cells were transfected with Mito-RFP and the indicated Em-Plin5 construct, and LDs labeled with Bodipy 665/676. Merged micrograph includes Mito-RFP and Bodipy 665/676 channels to demonstrate co-localization (white).

(B-C) Quantification of images from A. (B) Colocalization of LDs with mitochondria was measured as \% LD pixels overlapping with mitochondria, and (C) LD area/cell was measured, normalized to control.

(D-E) C2C12 were assayed as described in Fig. 2A-B, following RNAi treatment using nontargeting or Fatp4 siRNA. (D) Red C12 incorporation into LDs following CM chase was measured, normalized to control cells. (E) Red C12 accumulation into mitochondria relative to initial LD incorporation following HBSS starvation was measured, normalized to control cells. 
(F) Proposed model of FA trafficking during starvation. Under basal conditions Plin5 interacts with ATGL and CGI-58 leading to inhibition of lipolysis. During starvation: (1) PKA phosphorylates Plin5 at S155; (2) phosphorylation of Plin5 promotes lipolysis and release of FAs; (3) released FAs are channeled to mitochondria, where Fatp4 converts FAs to Acyl-CoAs allowing; (4) transport into mitochondria for $\beta$-oxidation.

Cartoon created with Biorender.com.

Scale bar, $10 \mu \mathrm{m}$. Data are expressed as means, error bars represent \pm SEM. ${ }^{*} p<0.05 .{ }^{* *} p<$ $0.01^{* * *} p<0.001$

\section{Supplemental Figure Legends}

\section{Supplemental Figure 1. Effect of Plin5 on LD-mito contacts and LD size in C2C12 myoblasts}

(A) Micrographs of $\mathrm{C} 2 \mathrm{C} 12$ myoblasts transfected with Mito-RFP and the indicated Em-Plin5 construct, and labeled for LDs with Bodipy 665/676. Merged micrograph includes Mito-RFP and Bodipy 665/667 channels to demonstrate co-localization (white).

(B-C) Quantification of images from (A). (B) Colocalization of LDs with mitochondria was measured as \% LD pixels overlapping with mitochondria, and (C) LD area/cell was measured, normalized to control.

(D) Quantification of LD mean speed in C2C12 myoblasts transfected with the indicated EmPlin5 construct, normalized to myoblasts transfected with Em control.

Scale bar, $10 \mu \mathrm{m}$. Data are expressed as means, error bars represent \pm SEM. ${ }^{* *} p<0.01,{ }^{* * *} p<$ 0.001 .

Supplemental Figure 2. Plin5 phosphomimetic constructs promote LD-mito contacts and do not alter Cpt1b expression in $\mathrm{C} 2 \mathrm{C} 12$ myoblasts

(A) Micrographs of C2C12 myoblasts transfected with Mito-RFP and indicated Em-Plin5 construct, and labeled for LDs with Bodipy 665/676. Merged micrograph includes Mito-RFP and Bodipy $665 / 667$ channels to demonstrate co-localization as shown by white pixels.

(B)Quantification of images from A. (B) Colocalization of LDs with mitochondria was measured as \% LD pixels overlapping with mitochondria.

(C) Quantitation of Cpt1b expression in C2C12 myoblasts 48 hrs after transfection with the indicated Em-Plin5 construct, normalized to myoblasts transfected with Em control.

Scale bar, $10 \mu \mathrm{m}$. Data are expressed as means \pm SEM. ${ }^{* *} p<0.01,{ }^{* * *} p<0.001$

Supplemental Figure 3. Plin5 and Fatp4 Pex constructs do not alter peroxisome area (A-C) Effect of organelle-targeted constructs on organelle area. (A) Quantitation of the effect of Em-Pex constructs on peroxisome area/cell, normalized to control. (B) Quantitation of the effect of Em-HP and TagBFP-Pex constructs on (B) LD and (C) peroxisome area/cell was measured, normalized to control.

Data are expressed as means, error bars represent \pm SEM.

\section{Supplemental Figure 4. Validation of Fatp4 RNAi}

(A-B) C2C12 myoblasts were transfected for $24 \mathrm{hr}$ with non-targeting or Fatp4 siRNA. Following RNAi, cells were transfected with Em or Em-Plin5 construct. (A) Representative western blot of Fatp4 abundance following RNAi. (B) Quantitation of Fatp4 expression, normalized to nontargeting siRNA treated cells.

Data are expressed as means, error bars represent \pm SEM. ${ }^{*} p<0.05,{ }^{* *} p<0.01$.

\section{Supplemental Movies}


(S1-S4) Airyscan movies of U2OS cells transfected with Mito-RFP (magenta) and labeled for LDs with Bodipy 665/676 (green). Cells were additionally transfected with (S1) Em, (S2) EmPlin5, (S3) Em-Plin5 (1-424) or (S4) Em-HP-Plin5 (425-463) (orange). Images were acquired every $27 \mathrm{~s}$ for 10 frames.

\section{Supplemental Table 1}

List of statistically significant Plin5-associate proteins identified in the LC-MS/MS based proteomics analysis. FC_A is enrichment relative to Em control, 2 was used as the cut off. SAINT Score is the probability a detected protein is a true interactor, 0.75 was used as the cutoff. Fold change is normalized to Plin5 abundance.

\section{Supplemental Table 2}

List of plasmids used in this study. 
A

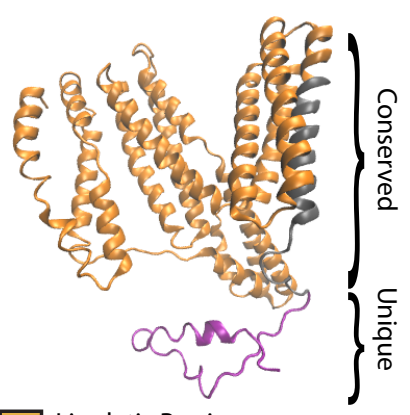

B

G

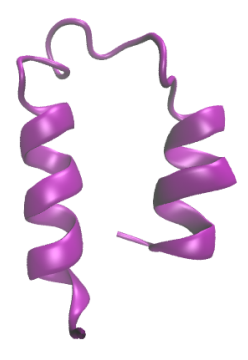

$$
\begin{aligned}
& \text { (1-395) } \\
& \text { (1-3arrier }
\end{aligned}
$$

Tether Domain

$$
\text { (425-463) }
$$

C

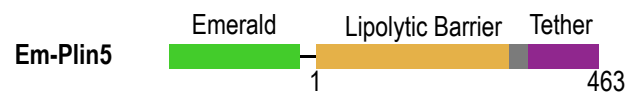

$$
\underset{(1-453)}{E m-P \operatorname{lin} 5 C \Delta}
$$

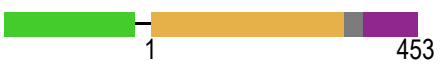

Em-Plin5C $\Delta$ (1-443)

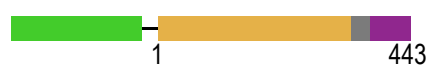

Em-Plin5C $\Delta$ (1-424)

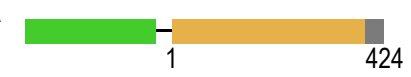

D
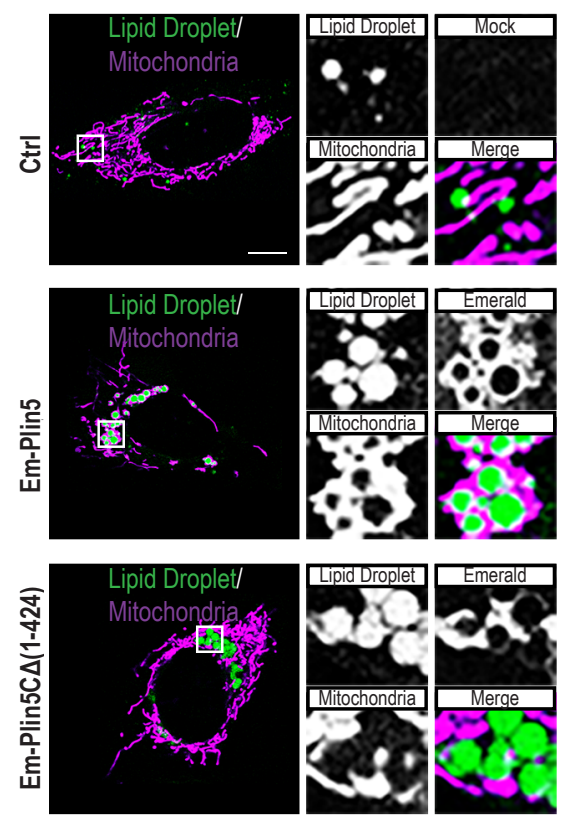

E

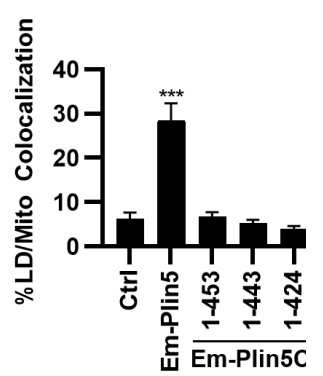

F

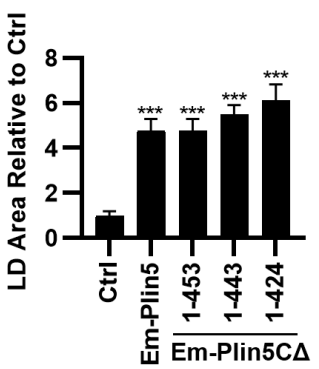

Em-Plin5

Em-HP-Plin5C
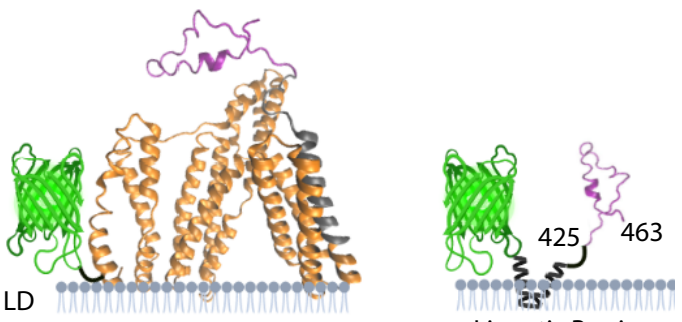

Lipoytic Barrier

replaced by Gpat4-HP

H

Em-HP

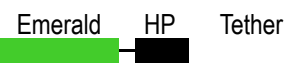

Em-HP-Plin5C (396-463)

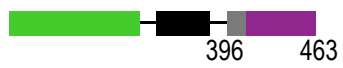

Em-HP-Plin5C (415-463)

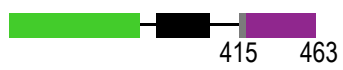

Em-HP-Plin5C (425-463)

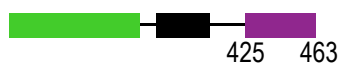

EM-HP-Plin5C (435-463)
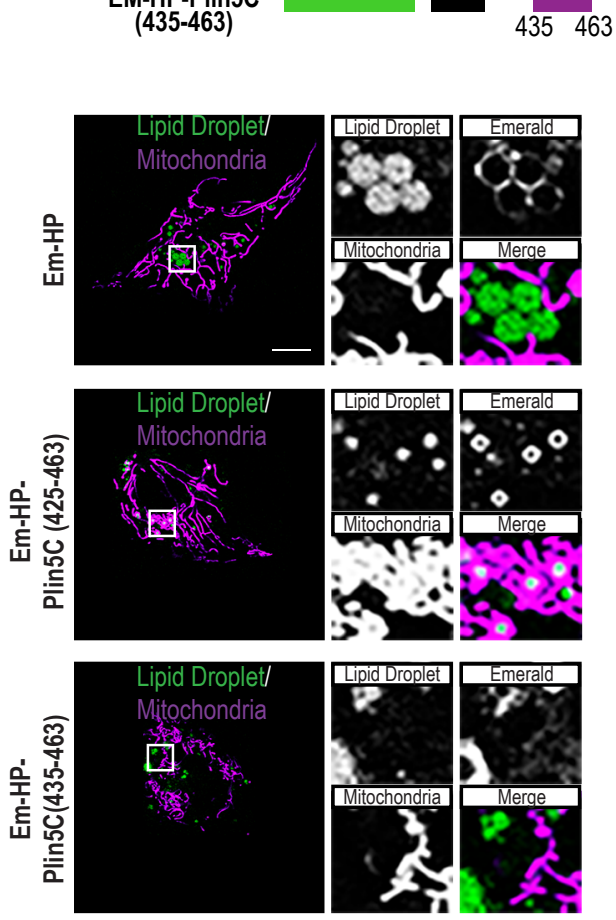

J

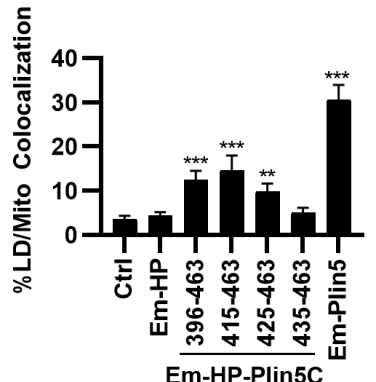

Figure 1

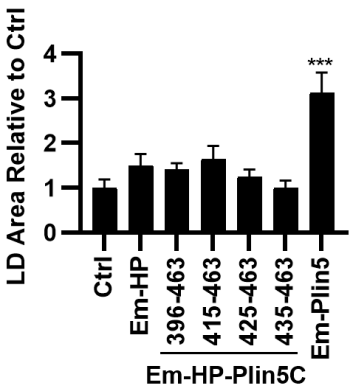


A

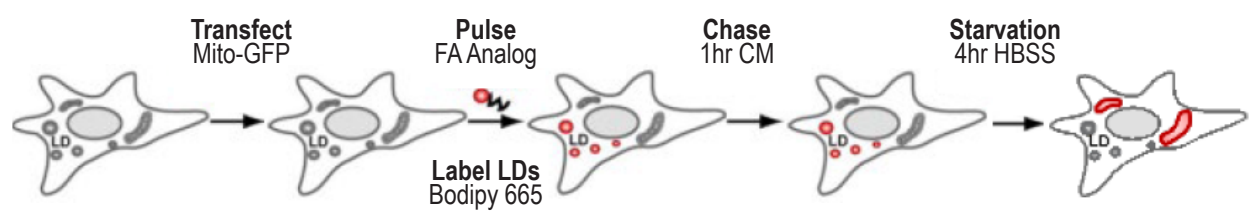

B

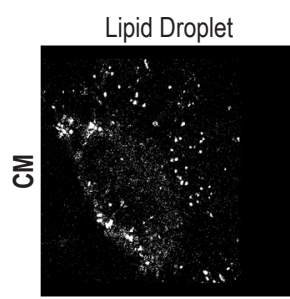

RedC12

Mitochondria

Lipid Droplet

RedC12

Mitochondria

RedC12
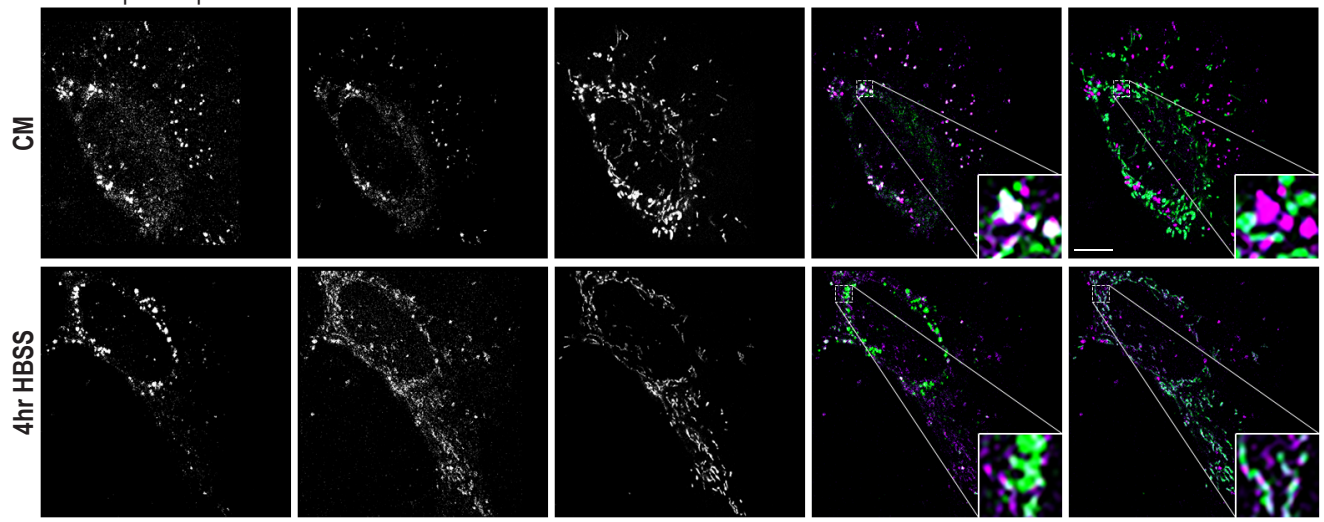

C

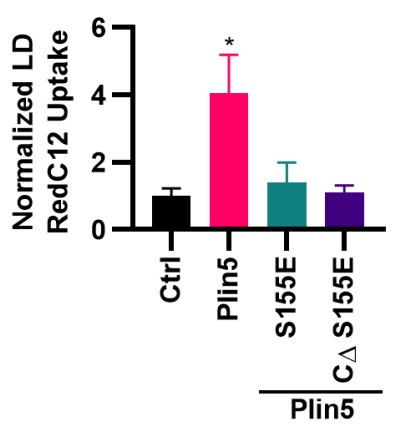

D

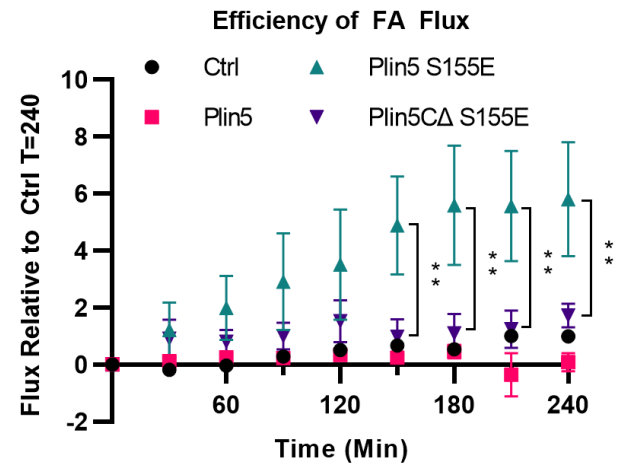

E

F
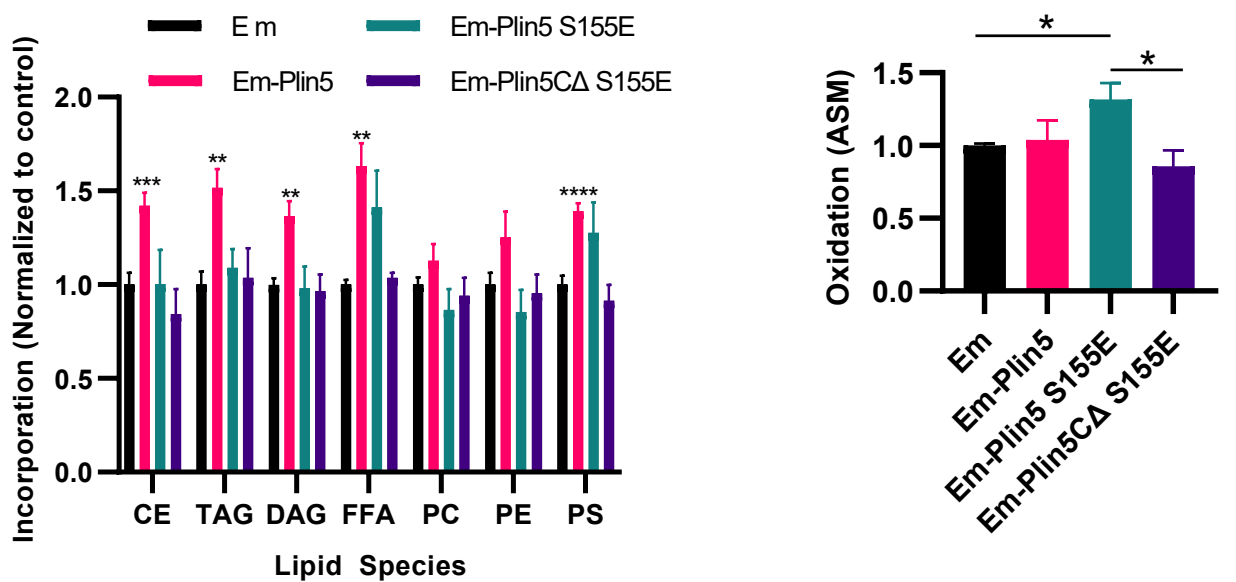
A Plin5C $\Delta$ 蒠离 PLIN5 ZNF598 。 TTC37 SACSO MMS19 $O$ Асот9ОО CPNE1 10 SH3BP4 $\bigcirc$ FATP4 $\bigcirc \bigcirc 0$ KIF20A ZNF281 NEDD1 FKBP8 ARHGEF40 SYNE2 KIAA1217 ASAP1

LIG1O ARHGEF1

LTN1 0

TRMT1L

PLCD3 $O$

PTCD3 0

GIT1 •

NormalizedFold Change

Relative Fold Change

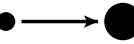

SAINT Score

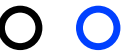

$\geq 0.9 \geq 0.75<0.75$
B

C

Mitochondrial

\begin{tabular}{|c|c|c|}
\hline \multirow{2}{*}{ Gene Name } & \multicolumn{2}{|c|}{ Fold Change (vs WT) } \\
\hline & $1-443$ & $1-424$ \\
\hline PLIN5 & 1.00 & 1.00 \\
\hline \multicolumn{3}{|c|}{ Fatty Acid Modifying } \\
\hline FATP4 & 0.833 & 0.343 \\
\hline ACOT9 & 0.715 & 0.152 \\
\hline \multicolumn{3}{|c|}{ Transcription } \\
\hline TASOR & 1.100 & 0.026 \\
\hline MMS19 & 0.715 & 0.205 \\
\hline KIF20A & 0.898 & 0.000 \\
\hline ZNF598 & 0.235 & 0.07 \\
\hline LIG1 & 1.660 & 0.036 \\
\hline SYNE2 & 1.287 & 0.118 \\
\hline PTCD3 & 2.617 & 0.326 \\
\hline TRMT1L & 1.782 & 0.015 \\
\hline \multicolumn{3}{|c|}{ Membrane Trafficking } \\
\hline ARHGEF1 & 1.682 & 0.061 \\
\hline SH3BP4 & 0.783 & 0.112 \\
\hline ARHGEF40 & 1.157 & 0.016 \\
\hline CPNE1 & 0.775 & 0.044 \\
\hline FKBP8 & 1.093 & 0.126 \\
\hline GIT1 & 3.559 & 0.032 \\
\hline ASAP1 & 1.417 & 0.282 \\
\hline \multicolumn{3}{|c|}{ Miscellaneous } \\
\hline TTC37 & 0.434 & 0.005 \\
\hline LTN1 & 1.754 & 0.115 \\
\hline SACS & 0.507 & 0.461 \\
\hline PLCD3 & 1.808 & 0.108 \\
\hline NEDD1 & 1.092 & 0.000 \\
\hline KIAA1217 & 1.407 & 0.040 \\
\hline
\end{tabular}

a-FATP4

a-MFN2
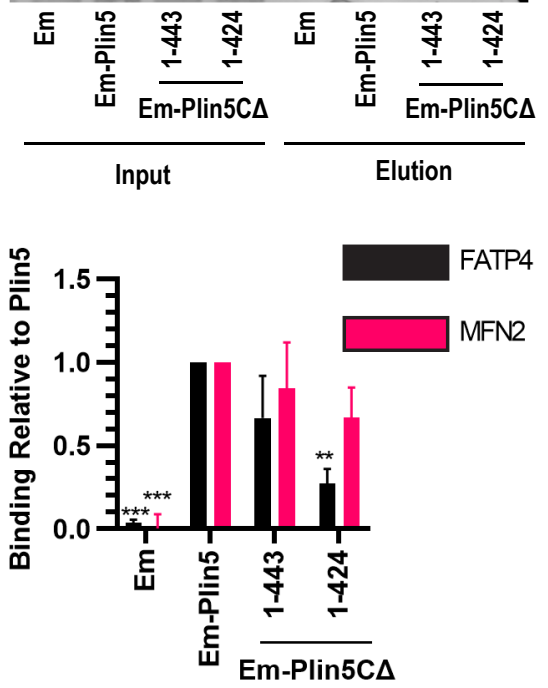
Pex-Em Pex Emerald Tether

Pex-Em-Plin5C $425 \quad 463$

C
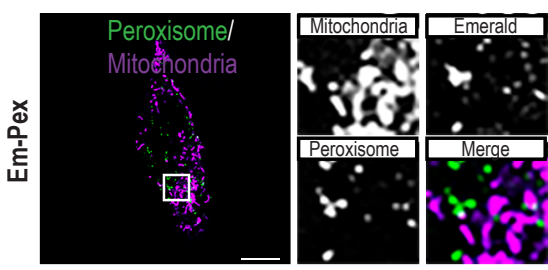

E

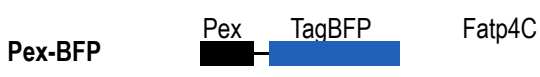

Pex-BFP-Fatp4C

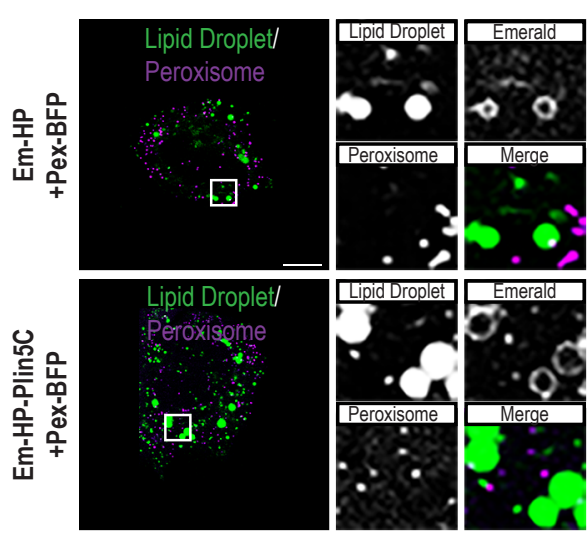

Perox

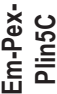

F

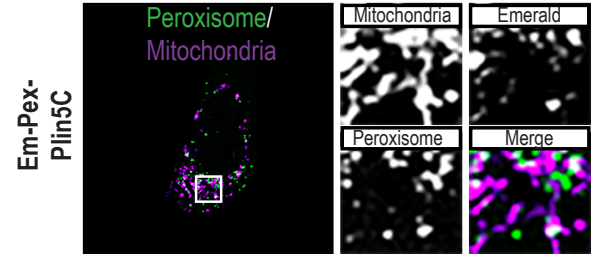

Fatp4 wild-type

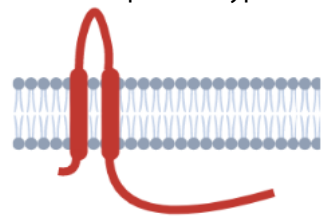

D

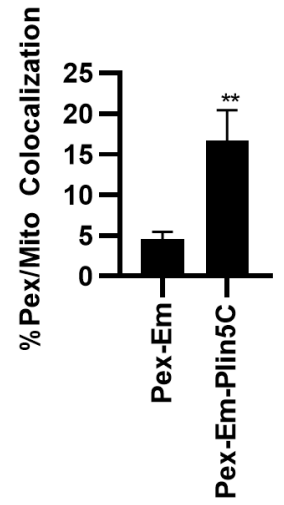

Pex-BFP-Fatp4C
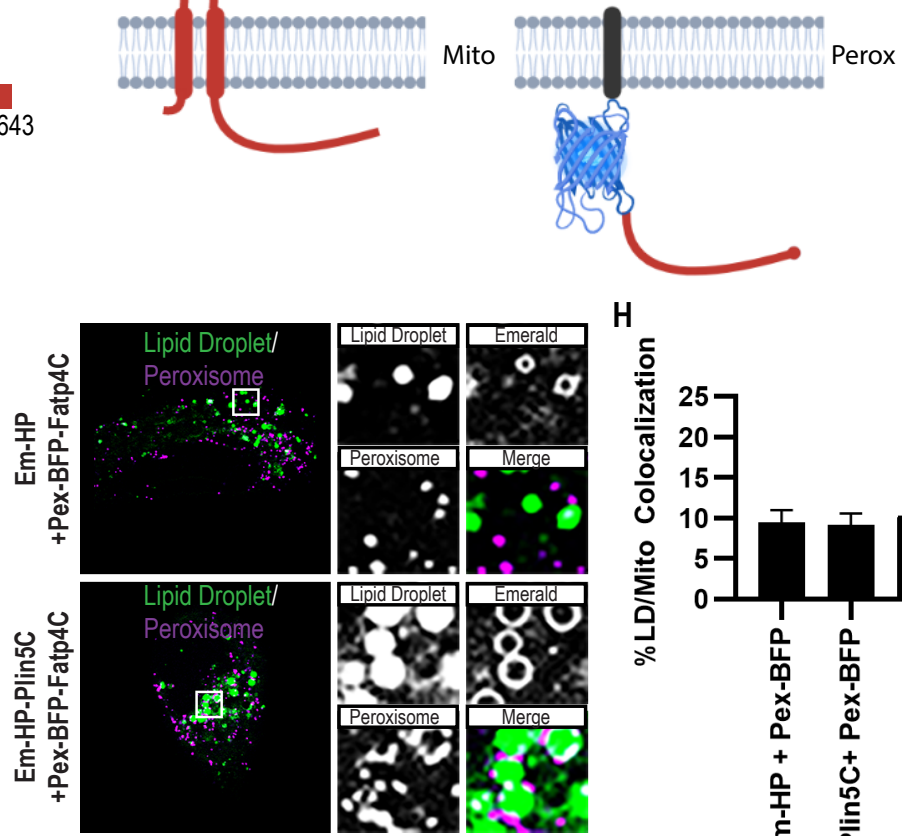

H
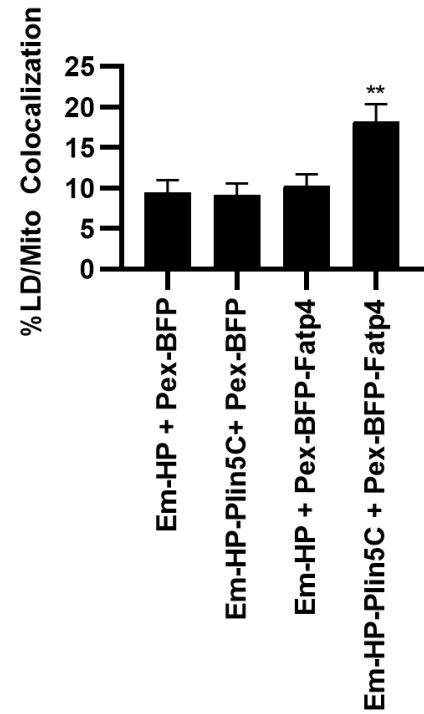
A
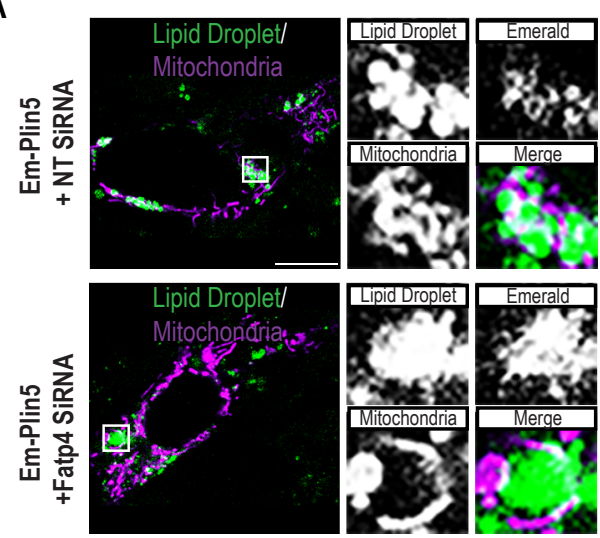

D
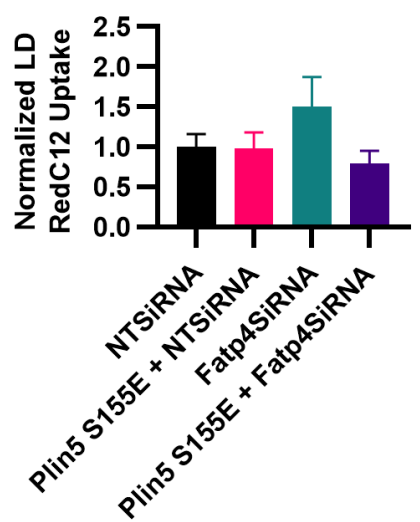

F

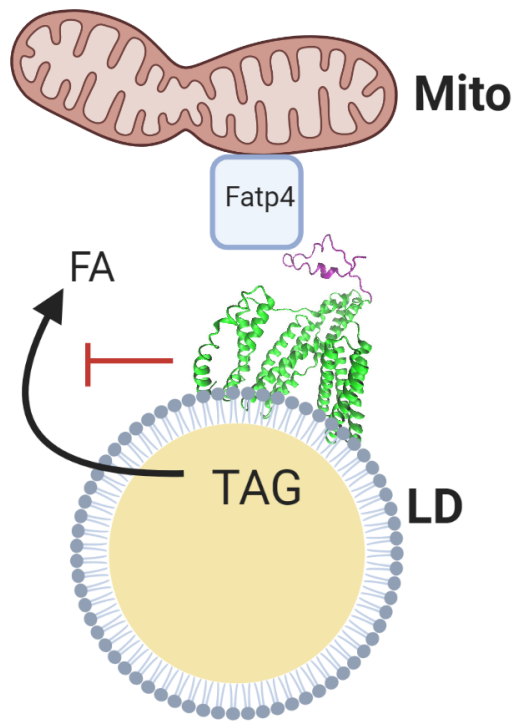

Basal
B

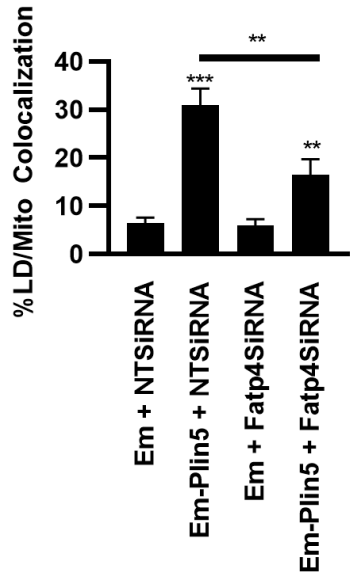

E

Efficiency of FA Flux
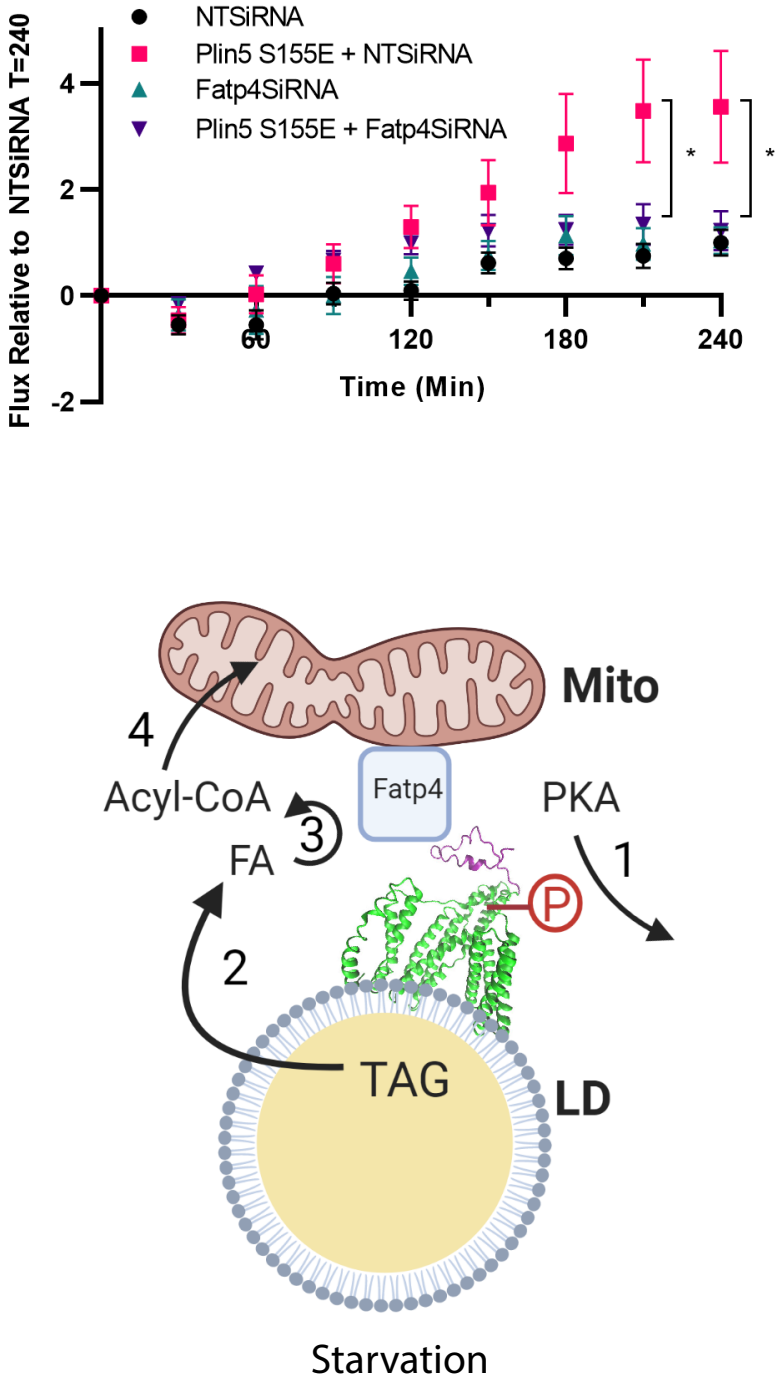

Figure 5 
A
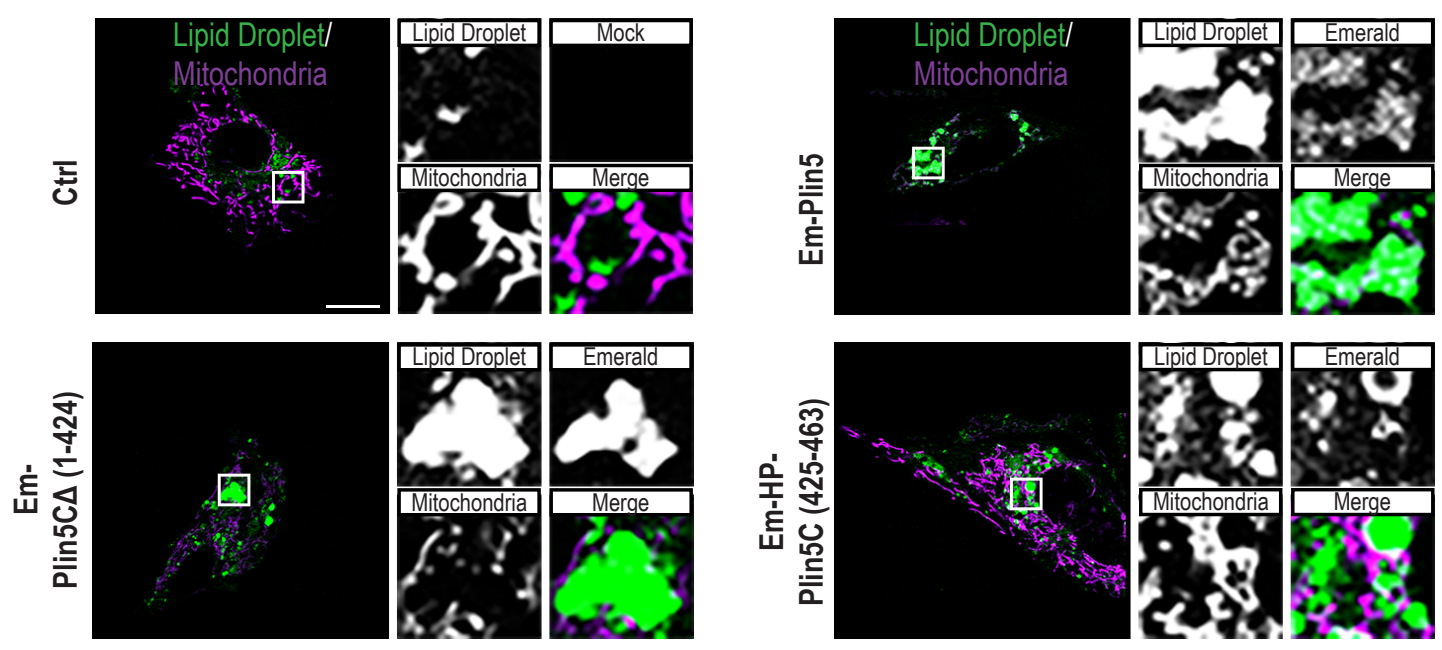

B

C

D
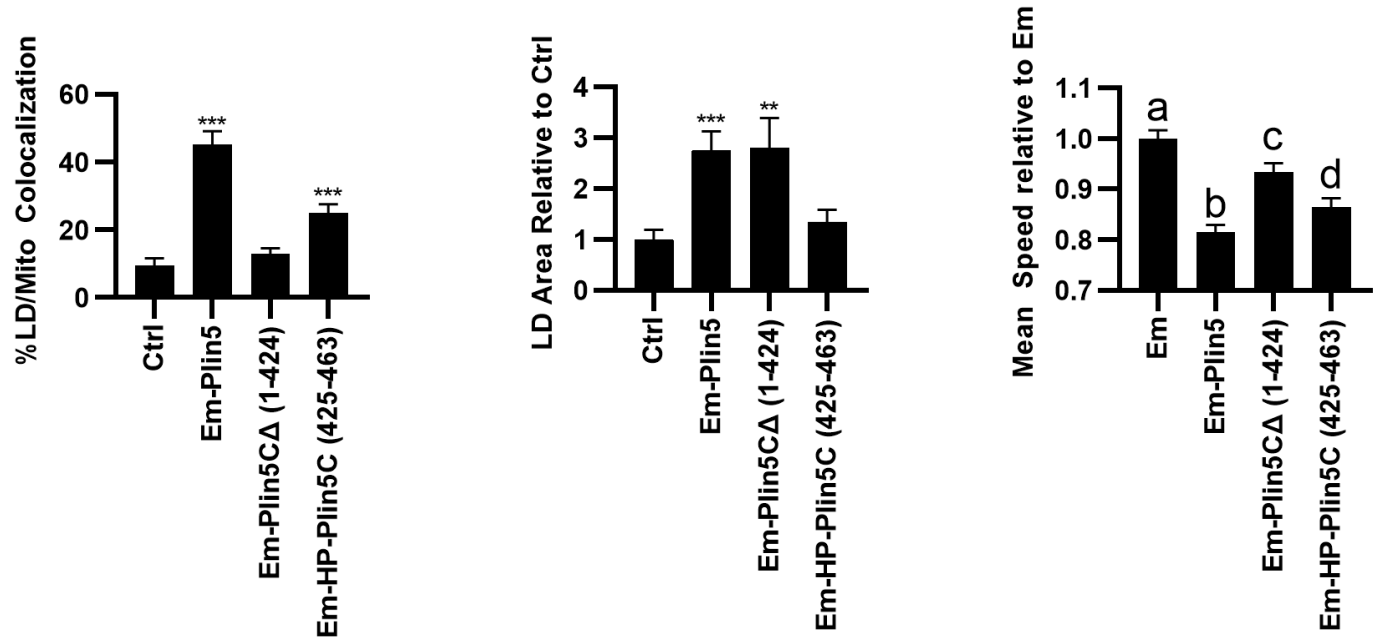

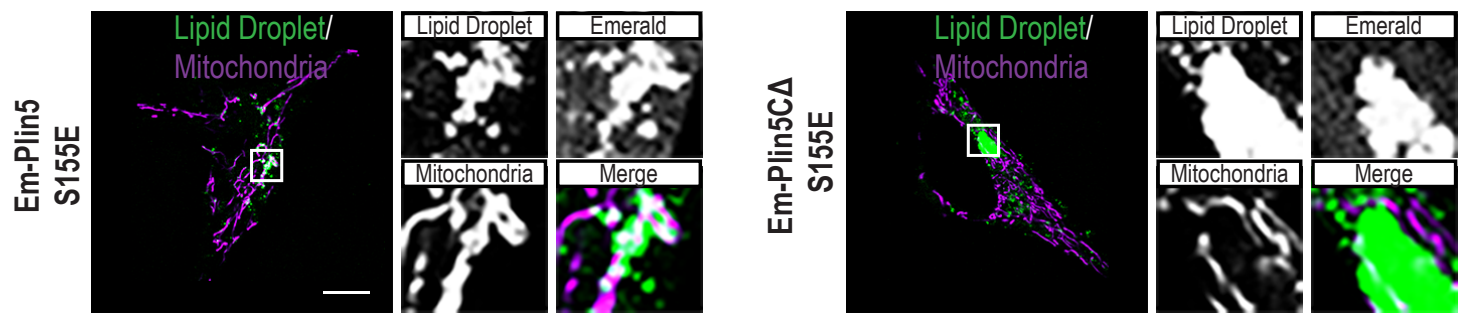

B

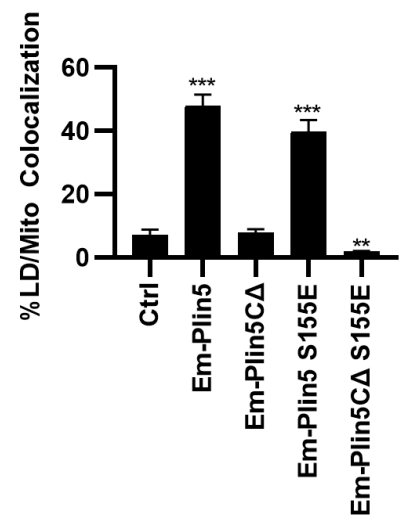

C

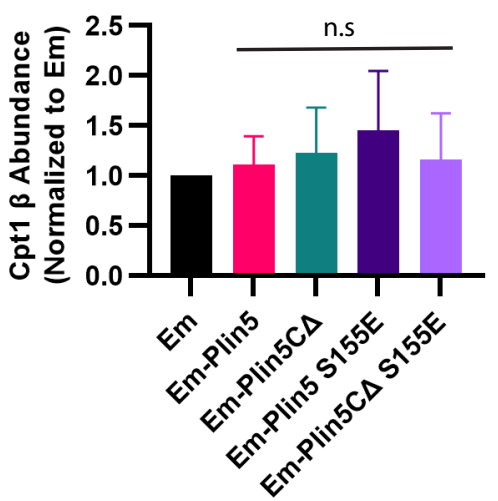


A

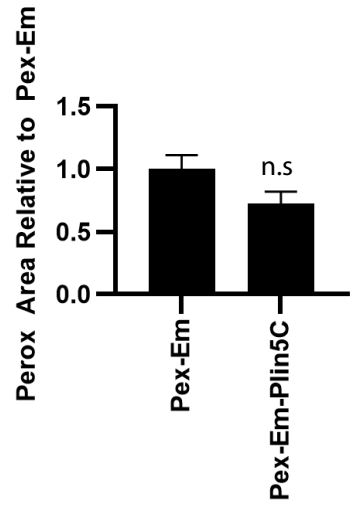

B

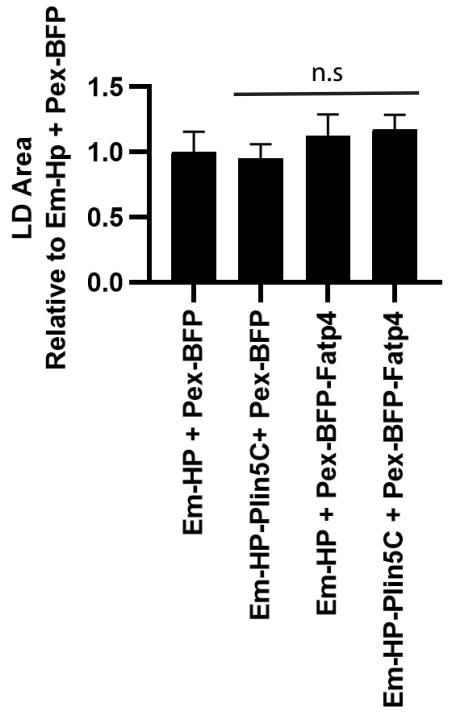

C

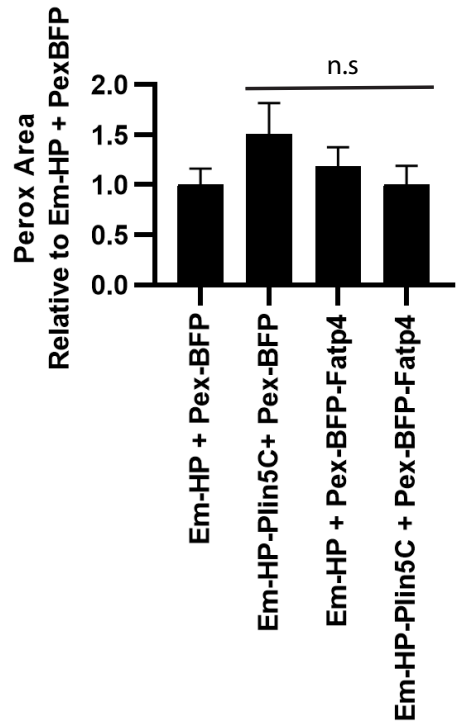



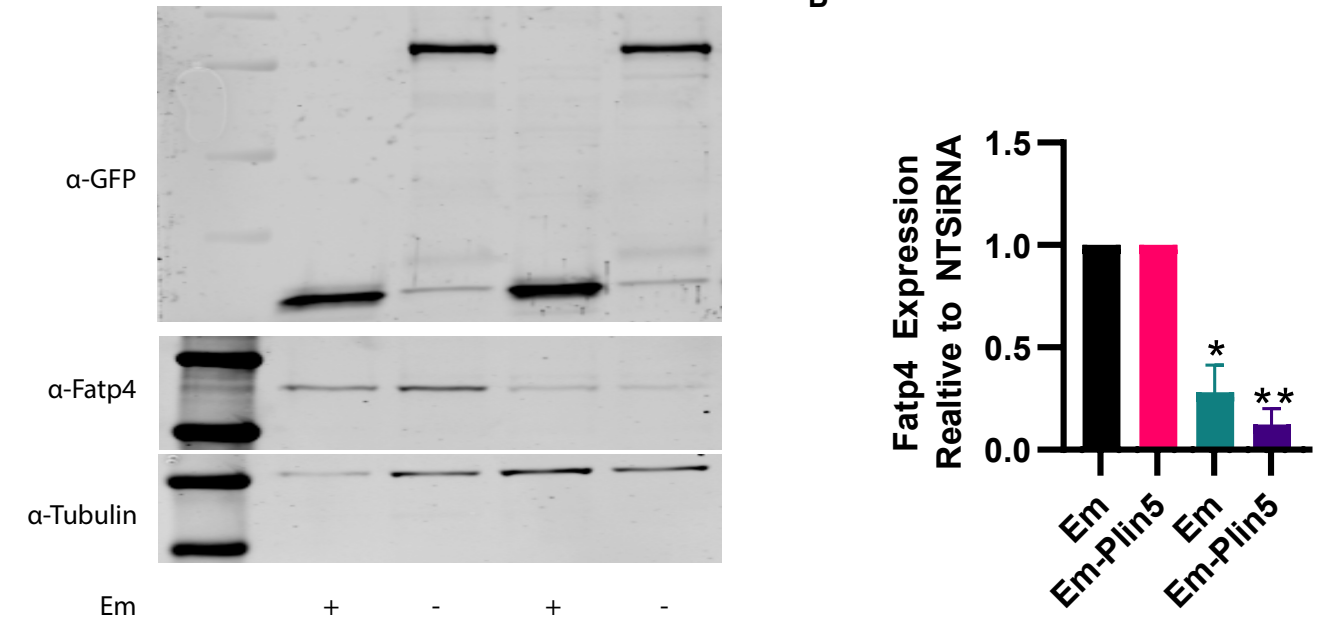

Em-Plin5

$\frac{-}{\text { NT SiRNA }} \quad \frac{-}{\text { Fatp4 SiRNA }}$

